\title{
Intrinsic Motivation Systems for Autonomous Mental Development
}

\author{
Pierre-Yves Oudeyer, Frédéric Kaplan, and Verena V. Hafner
}

\begin{abstract}
Exploratory activities seem to be intrinsically rewarding for children and crucial for their cognitive development. Can a machine be endowed with such an intrinsic motivation system? This is the question we study in this paper, presenting a number of computational systems that try to capture this drive towards novel or curious situations. After discussing related research coming from developmental psychology, neuroscience, developmental robotics, and active learning, this paper presents the mechanism of Intelligent Adaptive Curiosity, an intrinsic motivation system which pushes a robot towards situations in which it maximizes its learning progress. This drive makes the robot focus on situations which are neither too predictable nor too unpredictable, thus permitting autonomous mental development. The complexity of the robot's activities autonomously increases and complex developmental sequences self-organize without being constructed in a supervised manner. Two experiments are presented illustrating the stage-like organization emerging with this mechanism. In one of them, a physical robot is placed on a baby play mat with objects that it can learn to manipulate. Experimental results show that the robot first spends time in situations which are easy to learn, then shifts its attention progressively to situations of increasing difficulty, avoiding situations in which nothing can be learned. Finally, these various results are discussed in relation to more complex forms of behavioral organization and data coming from developmental psychology.
\end{abstract}

Index Terms-Active learning, autonomy, behavior, complexity, curiosity, development, developmental trajectory, epigenetic robotics, intrinsic motivation, learning, reinforcement learning, values.

\section{The Challenge of Autonomous Mental Development}

A LL humans develop in an autonomous open-ended manner through lifelong learning. So far, no robot has this capacity. Building such a robot is one of the greatest challenges to robotics today, and is the long-term goal of the growing field of developmental robotics [1], [2]. This paper explores a possible route towards such a goal. Our approach is inspired by developmental psychology and our ambition is to build systems featuring some of the fundamental aspects of an infant's development. More precisely, two remarkable properties of human infant development inspire us.

Manuscript received March 30, 2005; revised October 3, 2005. This work was supported in part by the ECAGENTS project founded by the Future and Emerging Technologies Program (IST-FET) of the European Community under EU R\&D Contract IST-2003-1940.

P.-Y. Oudeyer is with the Sony Computer Science Laboratory, Paris 6, rue Amyot 75005, Paris, France (e-mail: oudeyer@csl.sony.fr).

F. Kaplan is with CRAFT-Ecole Polytechnique Fédérale de Lausanne (EPFL), Lausanne CH-1015, Switzerland (e-mail: frederic.kaplan@epfl.ch).

V. V. Hafner is with the Sony Computer Science Laboratory Paris, Amyot 75005, Paris, France (e-mail: hafner@csl.sony.fr) and also with DAI Labor, 10587 Berlin, Germany (e-mail: vvh@ieee.org).

Color versions of one or more of the figures in this paper are available online at http://ieeexplore.ieee.org.

Digital Object Identifier 10.1109/TEVC.2006.890271

\section{A. Development is Progressive and Incremental}

First of all, development involves the progressive increase of the complexity of the activities of children with an associated increase of their capabilities. Moreover, infants' activities always have a complexity which is well-fitted to their current capabilities. Children undergo a developmental sequence during which each new skill is acquired only when associated cognitive and morphological structures are ready. For example, children first learn to roll over, then to crawl and sit, and only when these skills are operational, they begin to learn how to stand. Development is progressive and incremental. Taking inspiration from these observations, some roboticists argue that learning a given task could be made much easier for a robot if it followed a developmental sequence (e.g., "learning form easy mission" [3]). However, very often, the developmental sequence is crafted by hand: roboticists manually build simpler versions of a complex task and put the robot successively in versions of the task of increasing complexity. For example, if they want to teach a robot the grammar of a language, they first give it examples of very simple sentences with few words, and progressively they add new types of grammatical constructions and complications such as nested subordinates [4]. This technique is useful in many cases, but has shortcomings which limit our capacity to build robots that develop in an open-ended manner. Indeed, this is not practical. For each task that one wants the robot to learn, one has to design versions of this task of increasing complexity, and one also has to design manually a reward function dedicated to this particular task. This might be all right if one is interested in only one or two tasks, but a robot capable of lifelong learning should eventually be able to perform thousands of tasks, and even if one would engage in such a daunting task of manually designing thousands of specific reward functions, there is another limit. The robot is equipped with a learning machine whose learning biases are often not intuitive: this means that it is also conceptually difficult most of the time to think of simpler versions of a task that might help the robot. It is often the case that a task that one considers to be easier for a robot might turn out in fact to be more difficult.

\section{B. Development is Autonomous and Active}

This leads us to a second property of child development from which we should be inspired: it is autonomous and active. Of course, adults help by scaffolding children's environment, but this is just a help. Eventually, they decide by themselves what they do, what they are interested in, and what their learning situations are. They are not forced to learn the tasks suggested by adults, and they can invent their own. Thus, they construct by themselves their developmental sequence. Anyone who has ever played with an infant in its first year knows, for example, that it is extremely difficult to get the child to play with a toy that is 
chosen by the adult if other toys and objects are around. In fact, most often the toys that we think are adapted to them and will please them are not the ones they prefer. They can have much more fun and instructive play experiences with adult objects, such as magazines, keys, or flowers. Also, most of the time, infants engage in particular activities for their own sake, rather than as steps towards solving practical problems. This is indeed the essence of play. This suggests the existence of a kind of intrinsic motivation system, as proposed by psychologists like White [5], which provide internal rewards during these play experiences. Such internal rewards are obviously useful, since they are incentives to learn many skills that will potentially be readily available later on for challenges and tasks which are not yet foreseeable.

In order to develop in an open-ended manner, robots should certainly be equipped with capacities for autonomous and active development, and in particular with intrinsic motivation systems, forming the core of a system for task-independent learning. However, this crucial issue is still largely underinvestigated. The rest of this paper is organized in the following way. Section II presents a general discussion of research related to intrinsic motivation in the domain of psychology, neuroscience, developmental robotics, and active learning. Section III presents a critical review and a classification of existing intrinsic motivation systems and determines key characteristics important to permit autonomous mental development. Section IV describes in detail the algorithm of Intelligent Adaptive Curiosity (IAC). Section $\mathrm{V}$ discusses methodological issues for characterizing the behavior and performances of such systems. Section VI presents a first experiment using IAC with a simple simulated robot. Section VII presents a second more complex experiment involving a physical robot discovering affordances about entities in its environment. Section VIII discusses the results obtained in these two experiments in relation to more complex issues associated with behavioral organization and observation in infant development.

\section{BACKGROUND}

\section{A. Psychologyl}

White [5] presents an argumentation explaining why basic forms of motivation such as those related to the need for food, sex, or physical integrity maintenance cannot account for an animal's exploratory behavior, particularly in humans. He proposed rather that exploratory behaviors can be by themselves a source of rewards. Some experiments have been conducted showing that exploration for its own sake is an activity which is not always a secondary reinforcer, it is certainly a built-in primary reinforcer. The literature on education and development also stresses the distinction between intrinsic and extrinsic motivations [6]. Psychologists have proposed possible mechanisms which explain the kind of exploratory behavior that, for example, humans show. Berlyne [7] proposed that exploration might be triggered and rewarded for situations which include novelty, surprise, incongruity, and complexity. He also refined this idea by observing that the most rewarding situations

\footnotetext{
${ }^{1}$ The review of the psychology and neuroscience literature in this section is partly inspired from Barto et al. ([21]).
}

were those with an intermediate level of novelty, between already familiar and completely new situations. This theory has strong resonance points with the theory of flow developed by Csikszentmihalyi [8] which argues that a crucial source of internal rewards for humans is the self-engagement in activities which require skills just above their current level. Thus, for Csikszentmihalyi, exploratory behavior can be explained by an intrinsic motivation for reaching situations which represent a learning challenge. Internal rewards are provided when a situation which was previously not mastered becomes mastered within an amount of time and effort which must not be too small but also not too large. Indeed, in analogy with Berlyne [7], Csikszentmihalyi insists that the internal reward is maximal when the challenge is not too easy but also not too difficult.

\section{B. Neuroscience}

Recent discoveries showing a convergence between patterns of activity in the midbrain dopamine neurons and computational model of reinforcement learning have led to an important amount of speculations about learning activities in the brain [9]. Central to some of these models is the idea that dopamine cells report the error in predicting expected reward delivery. Most experiments in this domain focus on the involvement of dopamine for predicting extrinsic (or external) reward (e.g., food). Yet recently, some researchers provided ground for the idea that dopamine might also be involved in the processing of types of intrinsic motivation associated with novelty and exploration [10], [11]. In particular, some studies suggest that dopamine responses could be interpreted as reporting "prediction error" (and not only "reward prediction error") [12]. These findings support the idea that intrinsic motivation systems could be present in the brain in some form or another and that signals reporting prediction error could play a critical role in this context.

\section{Developmental Robotics}

Given this background, a way to implement an intrinsic motivation system might be to build a mechanism which can evaluate operationally the degree of "novelty," "surprise," "complexity," or "challenge" that different situations provide from the point of view of a learning robot, and then designing an associated reward ideally being maximal when these features are in an intermediate level, as proposed by Berlyne [7] and Csikszentmihalyi [13]. Autonomous and active exploratory behavior can then be achieved by acting so as to reach situations which maximize this internal reward. The challenge is to find a sensible manner to operationalize the concepts behind the words "novelty," "complexity," "surprise," or "challenge" which are only verbally described and often vaguely defined in the psychology literature.

Only a few researchers have suggested such implementations, and even fewer have tested them on real robots. Typically, they call these systems of autonomous and active exploratory behavior "artificial curiosity." Schmidhuber et al. [14], Thrun [15], and Herrmann et al. [16] provided initial implementations of artificial curiosity, but they did not integrate this concept within the problematic of developmental robotics, in the sense that they were not concerned with the emergent development sequence and with the increase of the complexity of their machines (and they did not use robots, but learning machines on some abstract 
problems). They were only concerned in how far artificial curiosity can speed up the acquisition of knowledge. The first integrated view of developmental robotics that incorporated a proposal for a novelty drive was described by Weng and colleagues [17], [18]. Then, Kaplan and Oudeyer proposed an implementation of artificial curiosity within a developmental framework [19], and Marshall et al., as well as Barto et al. presented variations on the novelty drive [20], [21]. As we will explain later on in this paper, these pioneering systems have a number of limitations making them impossible to use on real robots in real uncontrolled environments. Furthermore, to our knowledge, it has not yet been shown how they could successfully lead to the autonomous formation of a developmental sequence comprising more than one stage. This means that typically they have allowed for the development and emergence of one level of behavioral patterns, but did not show how new levels of more complex behavioral patterns could emerge without the intervention of a human or a change in the environment provoked by a human.

\section{Active Learning}

Interestingly, the mechanisms developed in these papers devoted to the implementation of artificial curiosity have strong similarities with mechanisms developed in the field of statistics, under the term "optimal experiment design" [22], and in machine learning, under the term "active learning" [23], [24]. In these contexts, the problem is summarized with the question: How to choose the next example for a learning machine in order to minimize the number of examples necessary to achieve a given level of performance in generalization? Or said another way: How to choose the next example so that the gain in information for the machine learner will be maximal? A number of techniques developed in active learning have been proven to speed up significantly the learning of machines (e.g., [25]-[31]) and even to allow performance on generalization which are not possible with passive learning [32]. Yet, these techniques were developed for applications in which the mapping to be learned was clean and typically presented as preprocessed well-prepared datasets. They are also typically based on mathematical theory like Optimal Experiment Design which assumes that the noise is independently normally distributed [33]. On the contrary, the domain that real robots shall investigate is the real unconstrained world, which is a highly complicated and "muddy" structure, as pointed out by Weng [34], full of very different kinds of intertwined non-Gaussian inhomogeneous noise. As a consequence, these methods cannot be used directly in the developmental robotics domain, and there is no obvious way to extend them in this direction. Moreover, there exists no efficient implementation for methods like optimal experiment design in continuous spaces, and already in discrete spaces the computational cost is high [35].

\section{Existing INTRINSIC Motivation Systems}

Existing computational approaches to intrinsic motivations and artificial curiosity are typically based on an architecture which comprises a machine which learns to anticipate the consequences of the robot's actions, and in which these actions are actively chosen according to some internal measures related to the novelty or predictability of the anticipated situation. Thus, the robots in these approaches can be described as having two modules: 1) one module implements a learning machine $\mathbf{M}$ which learns to predict the sensorimotor consequences when a given action is executed in a given sensorimotor context and 2) another module is a meta-learning machine metaM which learns to predict the errors that machine $\mathbf{M}$ makes in its predictions: these meta-predictions are then used as the basis of a measure of the potential interestingness of a given situation. The existing approaches can be divided into three groups, according to the way action-selection is made depending on the predictions of $\mathbf{M}$ and metaM.

\section{A. Group 1: Error Maximization}

In the first group (e.g., [15], [18], [20], and [21]) robots directly use the error predicted by metaM to choose which action to do. ${ }^{2}$ The action that they choose at each step is the one for which metaM predicts the largest error in prediction of $\mathbf{M}$. This has shown to be efficient when the machine $\mathbf{M}$ has to learn a mapping which is learnable, deterministic, and with homogeneous Gaussian noise [15], [17], [21], [32]. However, this method shows limitations when used in a real uncontrolled environment. Indeed, in such a case, the mapping that $\mathbf{M}$ has to learn is no longer deterministic, and the noise is vastly inhomogeneous. Practically, this means that a robot using this method will, for example, be stuck by white noise or, more generally, by situations which are inherently too complex for its learning machinery or situations for which the causal variables are not perceivable or observable by the robot. For example, a robot equipped with a drive which pushes it towards situations which are maximally unpredictable might discover and stay focused on movement sequences like running fast against a wall, the shock resulting in an unpredictable bounce (in principle, the bounce is predictable since it obeys the deterministic laws of classic mechanics but, in practice, this prediction requires the perfect knowledge of all the physical properties of the robot body, as well as those of the wall, which is typically far from being the case for a robot). So, in uncontrolled environments, a robot equipped with this intrinsic motivation system will get stuck and display behaviors which do not lead to development and that can sometimes even be dangerous.

\section{B. Group 2: Progress Maximization}

A second group of models tried to avoid getting stuck in the presence of pure noise or unlearnable situations by using more indirectly the prediction of the error of $\mathbf{M}$ (e.g., [16] and [19]). In these models, a third module that we call knowledge gain assessor (KGA) is added to the architecture. Fig. 1 shows an illustration of these systems. This new module enhances the capabilities of the meta-machine metaM: KGA predicts the mean error rate of $\mathbf{M}$ in the close future and in the next sensorimotor contexts. KGA also stores the recent mean error rate of $\mathbf{M}$ in the most recent sensorimotor contexts. The crucial point of these models is that the interestingness of candidate situations are evaluated using the difference between the expected mean error rate of the predictions of $\mathbf{M}$ in the close future, and the mean

\footnotetext{
${ }^{2}$ Of course, we are only talking about the "novelty" drive here: their robots are sometimes equipped with other competing drives or can respond to external human based reward sources.
} 


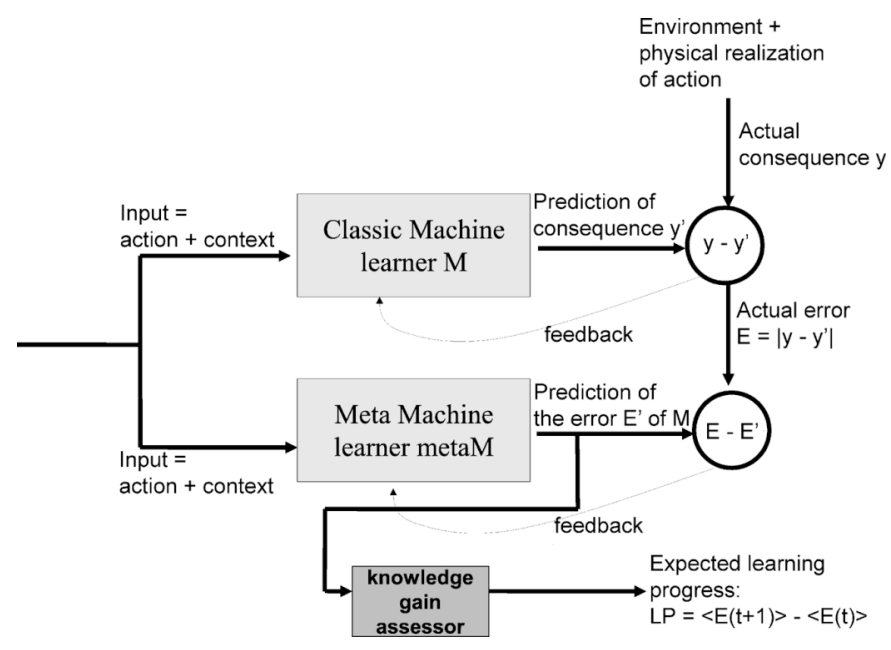

Fig. 1. The architecture used in various models of group 2 and group 3. Here, there is a module KGA which monitors the derivative of the errors of prediction of $\mathrm{M}$, which is the basis of an evaluation of learning progress. Some systems (group 2) evaluate the learning progress by measuring the decrease of the error rate of $\mathbf{M}$ in the close past, whatever the recent situations. Some other systems (group 3) evaluate the learning progress by measuring the decrease of the error rate of $\mathbf{M}$ in situations which are similar, but not necessarily close in time.

error rate in the close past. For each situation that the robot encounters, it is given an internal reward which is equal to the inverse of this difference (which also corresponds to the local derivative of the error rate curve of $\mathbf{M}$ ). This internal reward is positive when the error rate decreases, and negative when it increases. The motivation system of the robot is then a system in which the action chosen is that for which KGA predicts that it will lead to the greatest decrease of the mean error rate of $\mathbf{M}$. This ensures that the robot will not stay in front of white noise for a long time or in unlearnable situations because this does not lead to a decrease of its errors in prediction.

However, this method has only been tested in spaces in which the robot can do only one kind of activity, such as, for example, moving the head and learning to predict the position of high luminance points [19]. However, the ideal characteristic of a developmental robot is that it may engage in various kinds of activities, such as learning to walk, learning to grip things in its hand, learning to track a visual target, learning to catch the attention of other social beings, learning to vocalize, etc. In such cases, the robot can typically switch rapidly from one activity to the other, for example, making a trial at gripping an object that it sees and suddenly shifting to trying to track the movement of another being in its environment. In such a case, measuring the evolution in time of its performance in predicting what happens will lead to a measure which is hardly interpretable. Indeed, using the method we described in the last paragraph will make the robot compare its error rate in anticipation while it is trying to grip an object, with its error rate in anticipation while it is trying to anticipate the reaction of the other being when he vocalizes, if these two kinds of activities are sequenced. Thus, it will often lead the robot to compare its performances between activities which are of a different kind, which has no obvious meaning. And indeed, using this direct measure of the decrease in the error rate in prediction will provide the robot with internal rewards when shifting from an activity with a high mean error rate to activities with a lower mean error rate, which can be higher than the rewards corresponding to an effective increase of the skills of the robot in one of the activities. This will push the robot towards instable behavior, in which it focuses on the sudden shifts between different kinds of activities rather than concentrate on the actual activities.

\section{Group 3: Similarity-Based Progress Maximization}

Changes are needed so that methods based on the decrease of the error rate in prediction can still work in a realistic complex developmental robotics setup. It is necessary that the robot monitors the evolution of its error rate in prediction in situations which are of the same kind. It will no longer compare its current error rate with its error rate in the recent past, whatever the current situation and the situation in the close past are. The similarity between situations must be taken into account. Building a system which can do that correctly represents a big challenge. Indeed, a developmental robot will not be given an innate mechanism with a preprogrammed set of kinds of situations and a mechanism for categorizing each particular situation into one of these kinds. A developmental robot has to be able to build by itself a measure of the similarity of situations and ultimately an organization of the infinite continuous space of particular situations into higher level categories (or kinds) of situations. For example, a developmental robot does not know initially that on the one hand, there can be the "gripping objects" kind of activity and, on the other hand, the "vocalizing to others" kind of activity. Initially, the world is just a continuous stream of sensations and low-level motor commands for the robot.

A related approach, but with an active learning point of view rather than a developmental robotics point of view, was proposed in [14] and presented an implementation of the idea of evaluating the learning progress by monitoring the evolution of the error rate in similar situations. The implementation described was tested for discrete environments like a two-dimensional grid virtual world on which an agent could move and do one of four discrete actions. The similarity of two situations was evaluated by a binary function stating whether they correspond exactly to the same discrete state or not. From an active learning point of view, it was shown that in this case the system can significantly speed up the learning, even if some parts of the space are pure noise. This system was not studied under the developmental robotics point of view: it was not shown whether this allowed for a self-organization of the behavior of the robot into a developmental sequence featuring clearly several stages of increasing complexity. Moreover, because the system was only tested on a discrete simulated environment, it is difficult to generalize the results to real-world conditions with continuous environment and action spaces, and where two situations are never numerically exactly the same. Nevertheless, this paper suggests a possible manner to use this method in continuous spaces. It is based on the use of a learning machine such as a feedforward neural network which takes as input a particular situation and predicts the error associated with the anticipation of the consequence of a given action in this situation. This measure is then used in a formula to evaluate the learning progress. Thanks to the generalization properties of a machine like a neural network, the author claims that the mechanism will correctly generalize 
the evaluation of learning progress from one situation to similar situations. However, it is not clear how this will work in practice since the error function, and thus the learning progress function, are locally highly nonstationary. This provokes a risk of overgeneralization. Another limit of this work resides within the particular formula that is used to evaluate the learning progress associated with a candidate situation, which consists in making the difference between the error in the anticipation of this situation before it has been experienced and the error in the anticipation of exactly the same situation after it has been experienced. On the one hand, this can only work for a learning machine with a low learning rate, as pointed out by the author, and will not work with, for example, one-shot learning of memory-based methods. On the other hand, considering the state of the learning machine just before and just after one single experience can possibly be sensitive to stochastic fluctuations.

The next section will present a system which provides another implementation of the idea of evaluating the learning progress by comparing similar situations. This system is made to work in continuous spaces, and we will actually show that this system works both in a virtual robot setup and in a real robotic setup with continuous motor and/or perceptual spaces. One of its crucial features is that it introduces a mechanism of situation categorization, which splits the space incrementally and autonomously into different regions, which correspond to different kinds of activities from the point of view of the robot. This allows us to compare the similarity of two situations not directly based on their intrinsic metric distance, but on their belonging to a given situation category. Another feature is the fact that we monitor in each of these regions the evolution of the error rate in prediction for an extended period of time, which allows us to use smoothing procedures and avoid problems due to stochastic fluctuations. The "regional" evaluation of similarity combined with the smoothing of the error rate curve is a way to cope with the nonstationarity of the learning progress function. Another feature is that it makes no presupposition on the learning rate of the learning machines, and thus can be used with one-shot learning methods like nearest-neighbors algorithms, as well as with slowly learning neural networks for example.

\section{INTELLIGENT AdAPTIVE CURIOSITY (IAC)}

The system described in this section is called Intelligent Adaptive Curiosity (IAC).

- It is a motivation, or drive, in the same sense that food level maintenance or heat maintenance are drives, but instead of being about the maintenance of a physical variable, the IAC drive is about the maintenance of an abstract dynamic cognitive variable: the learning progress, which must be kept maximal. This definition makes it an intrinsic motivation.

- It is called curiosity because maximizing the learning progress pushes (as a side effect) the robot towards novel situations in which things can be learned.

- It is adaptive because the situations that are attractive change over time, indeed, once something is learned, it will not provide learning progress anymore.

- It is called intelligent because it keeps, as a side effect, the robot away both from situations which are too predictable and from situations which are too unpredictable (i.e., the edge of order and chaos in the cognitive dynamics). Indeed, thanks to the fact that one evaluates the learning progress by comparing situations which are similar and in a "regional" manner, the pathologic behaviors that we described in the previous section are avoided.

We will now describe how this system can be fully implemented. This implementation can be varied in many ways, for example, by replacing the implementation of the learning machines M, metaM, and KGA. The one we provide is basic and was developed for its practical efficiency. Also, it will be clear to the reader that in an efficient implementation, the machines M, metaM, and KGA are not easily separable (keeping them separate entities in the previous paragraphs was for reasons of keeping the explanation easier to understand).

\section{A. Summary}

IAC relies on a memory which stores all the experiences encountered by the robot in the form of vector exemplars. There is a mechanism which incrementally splits the sensorimotor space into regions, based on these exemplars. Each region is characterized by its exclusive set of exemplars and is also associated with its own learning machine, which we call an expert. This expert is trained with the exemplars available in its region. When a prediction corresponding to a given situation has to be made by the robot, then the expert of the region which covers this situation is picked up and used for the prediction. Each time an expert makes a prediction associated with an action which is actually executed, its error in prediction is measured and stored in a list which is associated to its region. Each region has its own list. This list is used to evaluate the potential learning progress that can be gained by going in a situation covered by its associated region. This is made based on a smoothing of the list of errors, and on an extrapolation of the derivative. When in a given situation, the robot creates a list of possible actions and chooses the one for which it evaluates, it will lead to a situation with maximal expected learning progress. ${ }^{3}$

\section{B. Sensorimotor Apparatus}

The robot has a number of real-valued sensors $s_{i}(t)$, which are here summarized by the vector $\mathbf{S}(\mathbf{t})$. Its actions are controlled by the setting of the real number values of a set of action/motor parameters $m_{i}(t)$, which we summarize using the vector $\mathbf{M}(\mathbf{t})$. These action parameters can potentially be very low level (for example, the speed of motors) or of a higher level (for example, the control parameters of motor primitives such as the biting or bashing movement that we will describe in the section devoted to the "Playground Experiment"). We denote the sensorimotor context $\mathbf{S M}(\mathbf{t})$ as the vector which summarizes the values of all the sensors and the action parameters at time $t$

\footnotetext{
${ }^{3} \mathrm{~A}$ variant of this system is the use of only one monolithic learning system, keeping the mechanism of region construction by incremental space splitting. In this case, for each prediction of the single learning system, its error is stored in the list corresponding to the region covering the associated situation. The evaluation of the expected learning progress of a candidate situation is the same as in the system presented here. However, we prefer to use one learning system per region in order to avoid forgetting problems which are typical of monolithic learning machines when used in a lifelong learning setup with various kinds of situations.
} 
[it is the concatenation of $\mathbf{S}(\mathbf{t})$ and $\mathbf{M}(\mathbf{t})$ ]. In all that follows, there is an internal clock in the robot which discretizes the time, and new actions are chosen at every time step.

\section{Regions}

IAC equips the robot with a memory of all the exemplars $(\mathbf{S M}(\mathbf{t}), \mathbf{S}(\mathbf{t}+\mathbf{1}))$ which have been encountered by the robot. There is a mechanism which incrementally splits the sensorimotor space into regions, based on these exemplars. Each region is characterized by its exclusive set of exemplars. At the beginning, there is only one region $\mathcal{R}_{1}$. Then, when a criterion $C_{1}$ is met, this region is split into two regions. This is done recursively. A very simple criterion $C_{1}$ can be used: when the number of exemplars associated with the region is above a threshold $T=250$, then split. This criterion allows us to guarantee a low number of exemplars in each leaf, which renders the prediction and learning mechanism that we will describe computationally efficient in the next paragraphs. The counterpart is that it will lead to systems with many regions which are not easily interpretable from a human point of view.

When a splitting has been decided, then another criterion $C_{2}$ must be used to find out how the region will be split. Again, the choice of this criterion was made so that it is computationally and experimentally efficient. The idea is that we split the set of exemplars into two sets so that the sum of the variances of $\mathbf{S}(\mathbf{t}+\mathbf{1})$ components of the exemplars of each set, weighted by the number of exemplars of each set, is minimal. Let us explain this mathematically. Let us denote

$$
\Gamma_{n}=\left\{(\mathbf{S M}(\mathbf{t}), \mathbf{S}(\mathbf{t}+\mathbf{1}))_{i}\right\}
$$

the set of exemplars possessed by region $\mathcal{R}_{n}$. Let us denote $j$ a cutting dimension and $v_{j}$ an associated cutting value. Then, the split of $\Gamma_{n}$ into $\Gamma_{n+1}$ and $\Gamma_{n+2}$ is done by choosing a $j$ and a $v_{j}$ such that (criterion $C_{2}$ ):

- all the exemplars $(\mathbf{S M}(\mathbf{t}), \mathbf{S}(\mathbf{t}+\mathbf{1}))_{i}$ of $\Gamma_{n+1}$ have the $j$ th component of their $\mathbf{S M}(\mathbf{t})$ smaller than $v_{j}$;

- all the exemplars $(\mathbf{S M}(\mathbf{t}), \mathbf{S}(\mathbf{t}+\mathbf{1}))_{i}$ of $\Gamma_{n+2}$ have the $j$ th component of their $\mathbf{S M}(\mathbf{t})$ greater than $v_{j}$;

- the quantity

$$
\begin{array}{r}
\left|\Gamma_{n+1}\right| \cdot \sigma\left(\left\{\mathbf{S}(\mathbf{t}+\mathbf{1}) \mid(\mathbf{S M}(\mathbf{t}), \mathbf{S}(\mathbf{t}+\mathbf{1})) \in \Gamma_{n+1}\right\}\right) \\
+\left|\Gamma_{n+2}\right| \cdot \sigma\left(\left\{\mathbf{S}(\mathbf{t}+\mathbf{1}) \mid(\mathbf{S M}(\mathbf{t}), \mathbf{S}(\mathbf{t}+\mathbf{1})) \in \Gamma_{n+2}\right\}\right)
\end{array}
$$

is minimal, where

$$
\sigma(\mathcal{S})=\frac{\sum_{v \in \mathcal{S}}\left\|v-\frac{\sum_{v \in \mathcal{S}} v}{|\mathcal{S}|}\right\|^{2}}{|\mathcal{S}|}
$$

where $\mathcal{S}$ is a set of vectors and $|\mathcal{S}|$ denotes the cardinal of $\mathcal{S}$.

Then, recursively and for each region, if the criterion $C_{1}$ is met, the region is split into two regions with the criterion $C_{2}$. This is illustrated in Fig. 2.

Each region stores all the cutting dimensions and the cutting values that were used in its generation, as well as in the generation of its parent experts. As a consequence, when a prediction has to be made of the consequences of $\mathbf{S M}(\mathbf{t})$, it is easy to find out the expert specialist for this case: it is the one for which $\operatorname{SM}(\mathbf{t})$ satisfies all the cutting tests (and there is always a single expert, which corresponds to each $\mathbf{S M}(\mathbf{t}))$.

\section{Experts}

To each region $\mathcal{R}_{n}$, there is an associated learning machine $\mathbf{E}_{\mathbf{n}}$, called an expert. A given expert $\mathbf{E}_{\mathbf{n}}$ is responsible for the prediction of $\mathbf{S}(\mathbf{t}+\mathbf{1})$ given $\mathbf{S M}(\mathbf{t})$ when $\mathbf{S M}(\mathbf{t})$ is a situation which is covered by its associated region $\mathcal{R}_{n}$. Each expert $\mathbf{E}_{\mathbf{n}}$ is trained on the set of exemplars which is possessed by its associated region $\mathcal{R}_{n}$. An expert can be a neural-network, a support-vector machine, or a Bayesian machine for example. For all learning machines whose training can be incremental, such as neural networks, support-vector machines, or memory-based methods, then the system is efficient since it is not necessary to retrain each expert on all the exemplars of each region, but just to update one single expert by feeding the new exemplar to it. Still, when a region is split, one cannot use directly the "parent" expert to implement the two children experts. Each child expert is typically a fresh expert retrained with the exemplars that its associated region has inherited. The computational cost associated with this is limited due to the fact that the number of exemplars is never higher than $T=250$ as guaranteed by the $C_{1}$ criterion. $^{4}$

\section{E. Evaluation of Learning Progress}

This partition of the sensorimotor space into different regions is the basis of our regional evaluation of learning progress. Each time an action is executed by the robot in a given sensorimotor context $\mathbf{S M}(\mathbf{t})$ covered by the region $\mathcal{R}_{n}$, the robot can measure the discrepancy between the sensory state $\widetilde{\mathbf{S}}(\mathbf{t}+\mathbf{1})$ that the expert $\mathbf{E}_{\mathbf{n}}$ predicted and the actual sensory state $\mathbf{S}(\mathbf{t}+\mathbf{1})$ that it

\footnotetext{
${ }^{4}$ Even computationally demanding learning machines such as nonlinear support vector machines require only a few dozens milliseconds on a standard computer to be trained with 250 examples, even if these examples have several hundred dimensions ([36]). In the experiments described in the next sections, we use a very simple learning algorithm for implementing the expert, the nearest-neighbors algorithm. In this case, there is not even a need for retraining the expert, since the expert is the set of exemplars. In general, the use of the nearest-neighbor algorithm is computationally costly when used at the prediction stage, since it requires as many computations of distances as there are exemplars. Again, the criterion $C_{1}$ guarantees that the number of exemplars is always low and allows for a fast computation of the closest exemplar. It is also interesting to note that if one would use a monolithic learning system with only one global expert, which is a variation of IAC mentioned earlier, then the use of the nearest-neighbors algorithm would soon become computationally very expensive since a lifelong learning robot can accumulate millions of exemplars. On the contrary, using local experts to which access is computed with a tree of cheap numerical comparisons (see Fig. 2) allows us to compute approximately correct global nearest neighbors with a logarithmic complexity $(O(\log (N)))$ rather than with a linear complexity $(O(n))$. In fact, using a tree structure with local experts not only allows to speed up the nearest-neighbors algorithm, but it also allows to increase the performances in generalization. In practice, this means that the system we present in this paper, when used, for example, with the nearest-neighbors algorithm, can update itself, as well as make predictions when it already possesses 3000000 exemplars in a few milliseconds on a personal computer, since in this case it requires about 17 scalar comparisons (depth of the corresponding balanced tree) and 250 distance computation between points. Admittedly, this requires a lot of memory, but it is interesting to note that the collection of 3000000 exemplars composed of, for example, 20 dimensions, which would take approximately 34 days in the case of the robots presented in the "Playground Experiment" section, would require about $230 \mathrm{Mb}$ in memory, which is much less than the capacity of most handheld computers nowadays.
} 


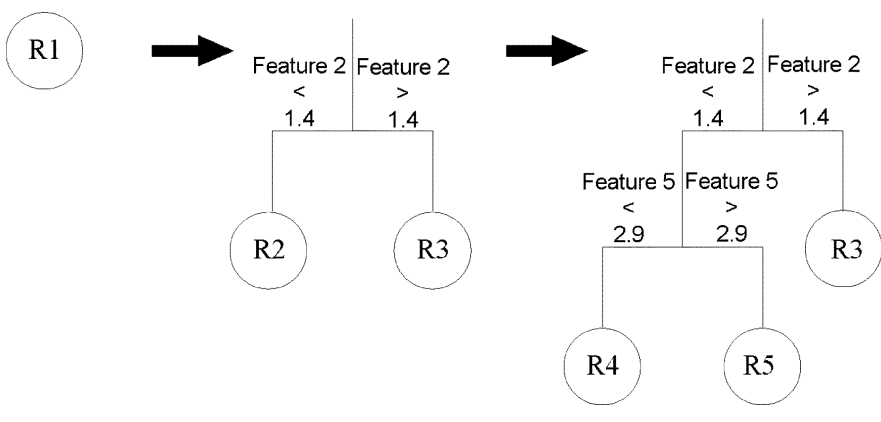

Fig. 2. The sensorimotor space is iteratively and recursively split into subspaces, which we call "regions." Each region $\mathcal{R}_{n}$ is responsible for monitoring the evolution of the error rate in the anticipation of the consequences of the robot's actions if the associated contexts are covered by this region. This list of regional error rates is used for learning progress evaluation.

measures. This provides a measure of the error of the prediction of $\mathbf{E}_{\mathbf{n}}$ at time $t+1$

$$
e_{n}(t+1)=\|\mathbf{S}(\mathbf{t}+\mathbf{1})-\widetilde{\mathbf{S}}(\mathbf{t}+\mathbf{1})\|^{2} .
$$

This squared error is added to the list of past squared errors of $\mathbf{E}_{\mathbf{n}}$, which are stored in association to the region $\mathcal{R}_{n}$. We denote this list

$$
e_{n}(t), e_{n}(t-1), e_{n}(t-2), \ldots, e_{n}(0) .
$$

Note that here $t$ denotes a time which is specific to the expert, and not to the robot, this means that $e_{n}(t-1)$ might correspond to the error made by the expert $\mathbf{E}_{\mathbf{n}}$ in an action performed at $t-10$ for the robot, and that no actions corresponding to this expert were performed by the robot since that time. These lists associated to the regions are then used to evaluate the learning progress that has been achieved after an action $\mathbf{M}(\mathbf{t})$ has been achieved in sensory context $\mathbf{S}(\mathbf{t})$, leading to a sensory context $\mathbf{S}(\mathbf{t}+\mathbf{1})$. The learning progress that has been achieved through the transition from the $\mathbf{S M}(\mathbf{t})$ context, covered by region $\mathcal{R}_{n}$, to the context with a perceptual vector $\mathbf{S}(\mathbf{t}+\mathbf{1})$ is computed as the smoothed derivative of the error curve of $\mathbf{E}_{\mathbf{n}}$ corresponding to the acquisition of its recent exemplars. Mathematically, the computation involves two steps.

- The mean error rate in prediction is computed at $t+1$ and $t+1-\tau$

$$
\begin{aligned}
\left\langle e_{n}(t+1)\right\rangle & =\frac{\sum_{i=0}^{\theta} e_{n}(t+1-i)}{\theta+1} \\
\left\langle e_{n}(t+1-\tau)\right\rangle & =\frac{\sum_{i=0}^{\theta} e_{n}(t+1-\tau-i)}{\theta+1}
\end{aligned}
$$

where $\tau$ is a time window parameter typically equal to 15 , and $\theta$ a smoothing parameter typically equal to 25 .

- The actual decrease in the mean error rate in prediction is defined as

$$
D(t+1)=<e_{n}(t+1)>-<e_{n}(t+1-\tau)>.
$$

We can then define the actual learning progress as

$$
L(t+1)=-D(t+1) .
$$

Eventually, when a region is split into two regions, both new regions inherit the list of past errors from their parent region, which allows them to make evaluation of learning progress right from the time of their creation.

\section{F. Action Selection}

We now have in place a prediction machinery and a mechanism which provides an internal reward (positive or negative)

$$
r(t)=L(t)
$$

each time an action is performed in a given context, depending on how much learning progress has been achieved. ${ }^{5}$ The goal of the intrinsically motivated robot is then to maximize the amount of internal reward that it gets. Mathematically, this can be formulated as the maximization of future expected rewards (i.e., maximization of the return), that is

$$
\left.E\left\{\sum_{t \geq t_{n}} \gamma^{t-t_{n}} r(t)\right)\right\}
$$

where $\gamma(0 \leq \gamma \leq 1)$ is the discount factor, which assigns less weigh on the reward expected in the far future.

This formulation corresponds to a reinforcement learning problem formulation [37], and thus the techniques developed in this field can be used to implement an action selection mechanism which will allow the robot to maximize future expected rewards efficiently. Indeed, in reinforcement learning models, a controller chooses which action $a$ to take in a context $s$ based on rewards provided by a critic. Traditional models view the critic as being external to the agent. Such situations correspond to extrinsically motivated forms of learning. But the critic can as well be part of the agent itself (as clearly argued by Sutton and Barto [37, pp. 51-54]). As a consequence, the algorithm described in this section can be interpreted as a critic capable of producing internal rewards $r(t)$ in order to guide the agent in its development. Thus, any existing reinforcement learning technique can be associated with the IAC drive.

One simple example would be to use Watkins' Q-learning [38]. The algorithm learns an action-value function $Q(s, a)$, estimating how good it is to perform a given action $a(\mathbf{M}(\mathbf{t})$ in our context) in a given contextual state $s(\mathbf{S}(\mathbf{t})$ in our context). "Good" actions are expected to lead to more future rewards (e.g., more future learning progress in our context). The algorithm can be described in the following procedural form:

- Initialize $Q(s, a)$ with small random uniform values;

- Repeat

- In situation $s$, choose $a$ using a policy derived from $Q$. For instance, choose $a$ that maximize $Q$ in most cases

\footnotetext{
${ }^{5}$ To integrate reward resulting from learning progress with other kinds of (possibly extrinsic) rewards, a weighted sum can be used. A parameter $\alpha_{i}$ specifies the relative weight of each reward type
}

$$
r(t)=\sum_{i} \alpha_{i} \cdot r_{i}(t)
$$


but every once in a while, with a probability $\epsilon$ instead select an action at random, uniformly (this is called an $\epsilon$-greedy action selection rule [37]);

- Perform action $a$, observe $r$, and the resulting state $s^{\prime}$;

$-Q(s, a) \leftarrow Q(s, a)+\alpha\left[r+\gamma \cdot \max _{a^{\prime}}\left(Q\left(s^{\prime}, a^{\prime}\right)\right)-\right.$ $Q(s, a)]$

$-s \leftarrow s^{\prime}$

where the parameter $\alpha$ is the learning rate controlling how fast the action-value function is updated by experience. Of course, all the complex issues traditionally encountered in reinforcement learning like tradeoff between exploration and exploitation stay crucial for systems using internal rewards based on intrinsic motivation.

The purpose of this paper is to focus on the study and understanding of the learning progress definition that we presented. Using a complex reinforcement machinery brings complexity and biases which are specific to a particular method, especially concerning the way they process delayed rewards. While using such a method with intrinsic motivation systems will surely be useful in the future, and is, in fact, an entire subject of research as illustrated by the work of Barto et al. [21] who have studied the use of sophisticated reinforcement learning techniques on a simple novelty-based intrinsic motivation system, we will now make a simplification which will allow us not to use such sophisticated reinforcement learning methods so that the results we will present in the experiment section can be interpreted more easily. Indeed, this is a necessary step since our intrinsic motivation system involves a nontrivial measure of learning progress which must be carefully understood. This simplification consists in having the system try to maximize only the expected reward it will receive at $t+1$, i.e., $E\{r(t+1)\}$. This permits us to avoid problems related to delayed rewards and it makes it possible to use a simple prediction system which can predict $r(t+1)$, and so evaluate $E\{r(t+1)\}$, and then be used in a straightforward action selection loop. The method we use to evaluate $E\{r(t+1)\}$ given a sensory context $\mathbf{S}(\mathbf{t})$ and a candidate action $\widehat{\mathbf{M}}(\mathbf{t})$, constituting a candidate sensorimotor context $\widetilde{\mathbf{S M}}(\mathrm{t})$ covered by region $\mathcal{R}_{n}$, is straightforward but revealed to be efficient, it is equal to the learning progress that was achieved in $\mathcal{R}_{n}$ with the acquisition of its recent exemplars, i.e.,

$$
E\{r(t+1)\} \approx L\left(t-\theta_{R_{n}}\right)
$$

where $t-\theta_{R_{n}}$ is the time corresponding to the last time region $\mathcal{R}_{n}$ and expert $\mathbf{E}_{\mathbf{n}}$ processed a new exemplar.

Based on this predictive mechanism, one can deduce a straightforward mechanism which manages action selection in order to maximize the expected reward at $t+1$.

- In a given sensory context $\mathbf{S}(\mathbf{t})$, the robot makes a list of the possible actions $\widetilde{\mathbf{M}}(\mathbf{t})$ which it can do; if this list is infinite, which is often the case since we work in continuous action spaces, a sample of candidate actions is generated.

- Each of these candidate actions $\widetilde{M}(\mathbf{t})$ associated with the context makes a candidate $\widetilde{\mathbf{S M}}(\mathbf{t})$ vector for which the robot finds out the corresponding region $\mathcal{R}_{n}$; then the formula we just described is used to evaluate the expected learning progress $E\{r(t+1)\}$ that might be the result of executing the candidate action $\widetilde{\mathbf{M}}(\mathbf{t})$.
- The action for which the system expects the maximal learning progress is chosen and executed except in some cases when a random action is selected $(\epsilon-$ greedy action selection rule). In the following experiments $\epsilon$ is typically 0.35 .

- After the action has been executed and the consequences measured, the system is updated.

\section{Methodological Issues for Measuring BEHAVIORAL COMPLEXITY}

From a developmental robotics point of view, intrinsic motivation systems are interesting as a way to achieve a continuous increase in behavioral complexity. This raises issues for finding adequate methods to evaluate such systems. Evaluation based on performance level for a set of predefined tasks is the most common way to assess the learning progress of adaptive robots. However, as intrinsic motivation systems are designed to result in task-independent autonomous development, using an evaluation paradigm coming from task-oriented design is not well adapted. Moreover, such evaluation methods are associated with the tempting anthropomorphic bias to evaluate how well robots manage to learn the tasks that humans can learn.

The issue is therefore to evaluate the increase of a robot's behavioral complexity during a developmental sequence. It is important to stress that there is not a single objective way for assessing the increase of complexity of a system. Complexity is always related to a given observer [39]. Three complementary approaches can be envisioned.

- First, it is possible to evaluate the increase in complexity from the robot's point of view. This means measuring internal variables that account for the open-endedness of its development (e.g., cumulative amount of learning progress, evolution of the performance of anticipations, and evolution of the way sensorimotor situations are categorized and represented).

- Second, behavioral complexity can be measured from an external point of view based on various complexity measures (information-theoretical measures such as the ones presented by Sporns and Pegors could be used in that respect [40]). The increase in behavioral complexity is assessed by pattern changes in these measures.

- Finally, the experimenter can adopt a method more similar to one used by a psychologist, interpreting developmental sequences as a set of successive stages. The stages of development introduced by Piaget are among the most famous examples of such qualitative descriptions [41]. Each transition between stages corresponds to a broad change in the structure or logic of children's intelligence and/or behavior. Based on clinical observations, dialogues, and small-scale experiments, the psychologist tries to interpret the signs of an internal reorganization. Therefore, the issue is to map external observations to a series of preexisting interpretative models. Transitions are most of the time progressive and cutting a developmental sequences into sharp division is usually difficult.

The following experiments will illustrate how a combination of some of these methods can be used to assess the development of a robot with an intrinsic motivation system. 


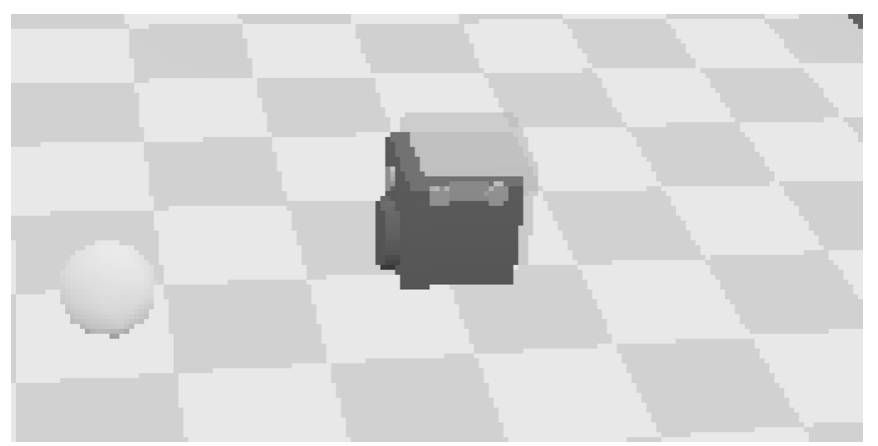

Fig. 3. The robotic setup. A two-wheeled robot moves in a room and there is also an intelligent toy (represented by a sphere) which moves according to the sounds that the robot produces. The robot perceives the distance between himself and the toy. The robot tries to predict this distance after performing a given action, which is a setting of (left wheel speed, right wheel speed, sound frequency). He chooses the actions for which it predicts its learning progress will be maximal.

\section{A First ExPERIMENT With a Simple SIMULATED RoBOT}

We present here a robotic simulation implemented with the Webots simulation software [42]. The purpose of this initial simulated experiment is to show and understand in detail the working of the IAC system in a continuous sensorimotor environment in which there are parts which are clearly inhomogeneous from the learning point of view: there is a part of the space which is easy to learn, a part of the space which contains more complex structures which can be learned, and a part of the space which is unlearnable.

\section{A. Motor Control}

The robot is a box with two wheels (see Fig. 3). Each wheel can be controlled by setting its speed (real number between -1 and 1). The robot can also emit a sound of a particular frequency. The action space is three-dimensional and continuous, and deciding for an action consists in setting the values of the motor vector $\mathbf{M}(\mathbf{t})$

$$
\mathbf{M}(\mathbf{t})=(l, r, f)
$$

where $l$ is the speed of the motor on the left, $r$ the speed of the motor on the right, and $f$ the frequency of the emitted sound. The robot moves in a room. There is a toy in this room that can also move. This toy moves randomly if the sound emitted by the robot has a frequency belonging to zone $f_{1}=[0 ; 0.33]$. It stops moving if the sound is in zone $f_{2}=[0,34 ; 0,66]$. The toy jumps into the robot if the sound is in zone $f_{3}=[0,67 ; 1]$.

\section{B. Perception}

The robot perceives the distance to the toy with simulated infrared sensors, so its sensory vector $\mathbf{S}(\mathbf{t})$ is one-dimensional

$$
\mathbf{S}(\mathbf{t})=(d)
$$

where $d$ is the distance between the robot and the toy at time $t$.

\section{Action Perception Loop}

As a consequence, the mapping that the robot is trying to learn is

$$
f: \mathbf{S M}(\mathbf{t})=(l, r, f, d) \longmapsto \mathbf{S}(\mathbf{t}+\mathbf{1})=(\widetilde{d}) .
$$

Using the IAC algorithm, the robot will thus act in order to maximize its learning progress in terms of predicting the next toy distance. The robot has no prior knowledge and, in particular, it does not know that there is a qualitative difference between setting the speed of the wheels and setting the sound frequency (for the robot, these are unlabeled motor channels). It does not know that there are three zones of the sensorymotor space of different complexities: the zone corresponding to sounds in $f_{1}$, where the distance to the toy cannot be predicted since its movement is random; the zone with sounds in $f_{3}$, where the distance to the toy is easy to learn and predict (it is always 0 plus a noise component because Webots simulates the imprecision of sensors and actuators); and the zone with sounds in $f_{2}$, where the distance to the toy is predictable (and learnable) but complex and dependant of the setting of the wheel speeds.

However, we will now show that the robot manages to autonomously discover these three zones, evaluate their relative complexity, and exploit this information for organizing its own behavior.

\section{Results}

First of all, one can study the behavior of the robot during a simulation from an external point of view. A way to do that is to use our knowledge of the structure of the environment in which the robot lives and build corresponding relevant measures characterizing the behavior of the robot within a given period of time: 1) the frequency of situations in which it emits a sound within $\left.f_{1} ; 2\right)$ the frequency of situations in which it emits a sound within $f_{2}$; and 3 ) the frequency of situations in which it emits a sound within $f_{3}$. Fig. 4 shows the evolution of these measures for 5000 time steps. Several phases can be identified.

- Stage 1: Initially, the robot produces all kinds of actions with a uniform probability, and in particular produces sounds with frequencies within the whole $[0,1]$ spectrum.

- Stage 2: After the first 250 first steps, the robot concentrates on emitting sounds within $f_{3}$, and emits sounds with frequencies within $f_{1}$ or $f_{2}$ very rarely.

- Stage 3: There is then a phase within which the robot concentrates on emitting sounds within $f_{2}$, and emits sounds with frequencies within $f_{1}$ or $f_{3}$ very rarely.

This shows that the robot consistently avoids the situations in which nothing can be learned, begins by easy situations, and then shifts autonomously to a more complex situation.

We can now study what happens from the robot's point of view. Fig. 5 shows a representation of the successive values of $\left\langle e_{n}(t)\right\rangle$ for all the regions $\mathcal{R}_{n}$ constructed by the robot at a given time $t$. As the time is here defined internally as the number of action selection loops, it corresponds to the number of actions that have been chosen by the robot, and to the number of exemplars that have been provided to it. The graph appears as a tree, which corresponds to the successive splitting of the space 


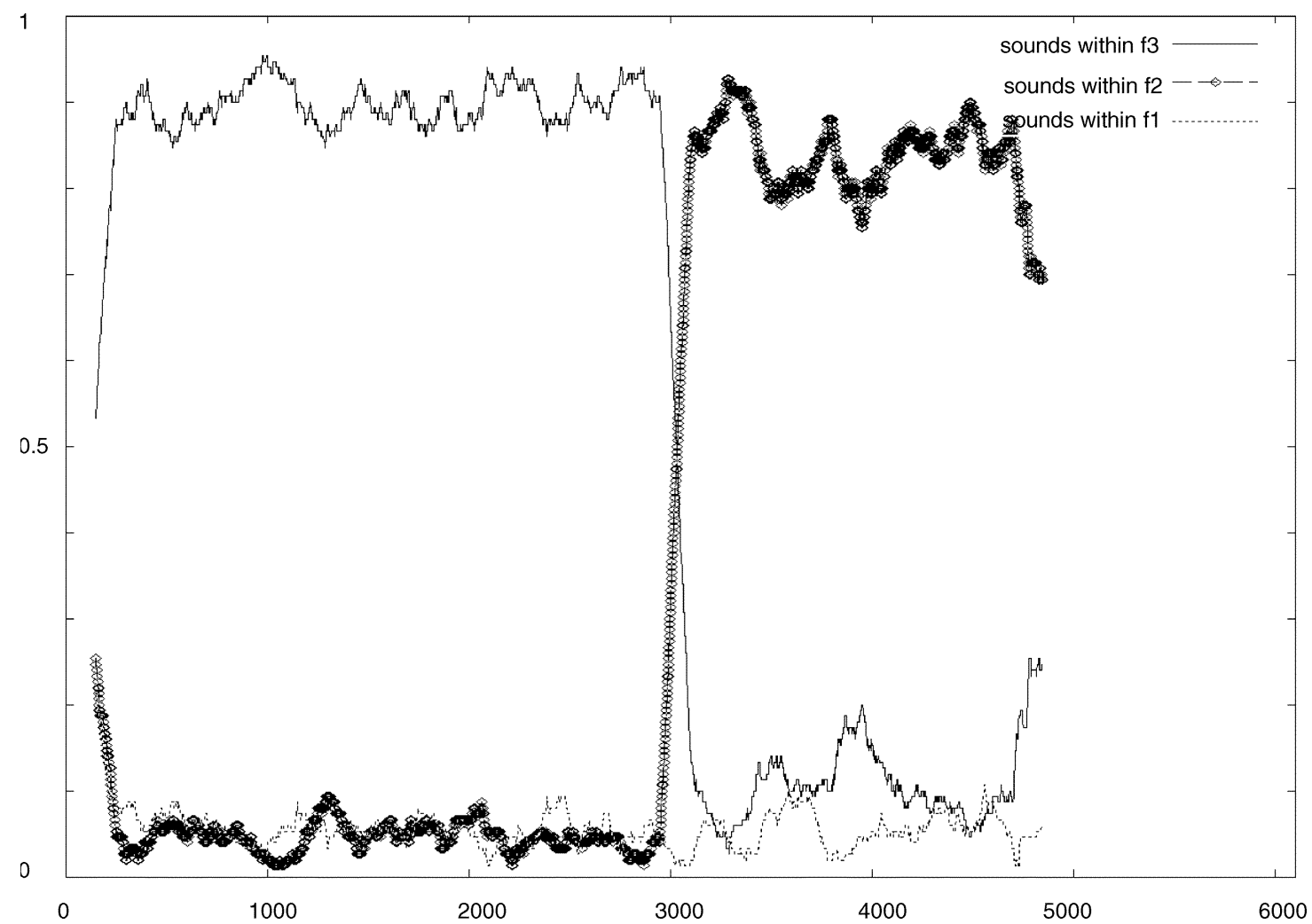

Fig. 4. Evolution of the percentage of time spent in: 1) situations in which the emitted sounds have a frequency within $f_{3}$ (continuous line); 2) situations in which the emitted sounds have a frequency within $f_{2}$ (dotted line); and 3) situations in which the emitted sounds have a frequency within $f_{1}$ (dashed line).

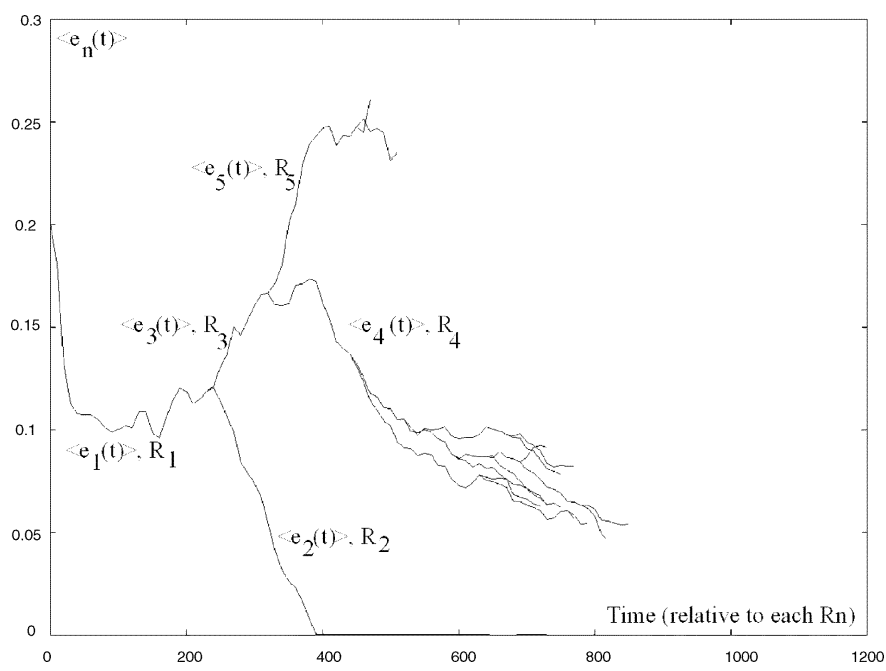

Fig. 5. Evolution of the successive values of $\left\langle e_{n}(t)\right\rangle$ for all the regions $\mathcal{R}_{n}$ constructed by the robot.

into regions. For example, between $t=0$ and $t=250$, there is only one curve because during that time there was only one region $\mathcal{R}_{1}$. This initial curve is the sequence of values of $\left\langle e_{1}(t)\right\rangle$. Then, because the criterion $C_{1}$ was met, this region splits into two regions $\mathcal{R}_{2}$ and $\mathcal{R}_{3}$, which also splits the curve into two curves, one corresponding to the successive values of $\left\langle e_{2}(t)\right\rangle$ and the other corresponding to the successive values of $\left\langle e_{3}(t)\right\rangle$. Then, the curves split again when their associated regions split, etc.
By looking at the trace of the simulation and the definitions of the regions associated to each curve, it is possible to figure out what the regions which are iteratively created look like. It appears that the first split appearing at $t=250$ corresponds to a split between situations in which the robot emits sounds with a frequency within $f_{3}\left(\mathcal{R}_{2}\right.$ on the graph), and situations in which the robot emits sounds with a frequency within $f_{2}$ or $f_{1}\left(\mathcal{R}_{3}\right.$ on the graph). To be exact, the system made a split by using the third dimension of $\mathbf{S M}(\mathbf{t})$, i.e., the frequency $f$, and using the cut value 0.35 , which means that the region $\mathcal{R}_{2}$ includes possibly a small portion of situations with a sound in $f_{2}$, since $f_{2}$ begins at $0.34 .{ }^{6}$ Now, we observe that the curve corresponding to $\mathcal{R}_{2}$ shows a sharp decrease in its error rate, while the curve in $\mathcal{R}_{3}$ shows an increase in the error rate. This explains why during this period, the robot will emit sounds with frequencies within $f_{3}$ : indeed, this corresponds to situations which are internally evaluated as providing the highest amount of learning progress at this time of its development. Nevertheless, as the robot sometimes does some random actions, the region $\mathcal{R}_{3}$ accumulates some more exemplars, and we observe that around $t=320$, it splits into $\mathcal{R}_{4}$ and $\mathcal{R}_{5}$. Looking at the trace shows that $\mathcal{R}_{4}$ corresponds to situations with sounds within $f_{2}$ and $\mathcal{R}_{5}$ with sounds within $f_{1}$. We observe that the error rate continues to increase until a plateau is reached for $\mathcal{R}_{5}$, while it begins to decrease for $\mathcal{R}_{4}$. During that time, the robot finally predicts

${ }^{6}$ This also shows that the splitting criteria $C_{1}$ and $C_{2}$ that we presented operate efficiently, since the system finds out by itself that this is the $f$ dimension which is the most relevant for cutting the space at the beginning of the development 
perfectly well situations with sounds with a frequency within $f_{3}$ and associated with $\mathcal{R}_{2}$ (it still takes a while because of the noise), and a plateau close to 0 in the error rate is reached. This is why at some point the robot shifts to situations in which it emits sounds with frequencies within $f_{2}$, which are situations which are a higher source of learning progress at this point in its development. The robot then tries to vary its motor speeds within this subspace with sounds with frequencies in $f_{2}$, learning to predict how these speeds affect the distance to the toy. The accumulation of new exemplars pushes the robot to split $\mathcal{R}_{4}$ into more regions, which is a refinement of its categorization of this kind of situations. Now, the system splits the space using the $l$ and $r$ dimensions, and the robot figures out how to efficiently explore the subspace of situations with sounds with frequencies within $f_{2}$, in terms of learning progress.

\section{E. Performance in Terms of Active Learning}

The efficiency of the exploration of this subspace of situations with sounds in $f_{2}$, where interesting things can be learned, can be evaluated if we reformulate IAC within the problematics of active learning. This will also allow us to evaluate the efficiency of the IAC algorithm from the point of view of active learning. Indeed, as we explained in the introduction, in the field of machine learning and data mining, the search for methods which allow us to reduce the number of examples needed to achieve a given level of performance in generalization for a machine which learns an input-output mapping, is of growing interest (here, the input is $\mathbf{S M}(\mathbf{t})$ and the output is $S M(t+1)$ ). While IAC was designed as a system for driving the development of a robot, it can also be considered as a pure active learning algorithm, and in this respect, it is interesting to evaluate how it compares with standard existing algorithms. Thus, we will use two reference algorithms to evaluate the performance of IAC. The first one follows the most common idea in the field of active learning [15], [24], [25]. The next action (also called query or experiment depending on the authors) is chosen so that it corresponds an input-output pair for which the machine evaluates that its prediction for this pair will be maximally false as compared with its prediction for possible other pairs. It is easy to adapt this idea using the same algorithmic architecture than the one used for IAC: when the robot has to decide for an action in a given context, it makes the list of possible actions within that context, then for each of them evaluates the expected error in prediction using the quantity $\left\langle e_{\text {mean }}(t)\right\rangle$ defined earlier, and finally chooses the action for which this quantity is maximal. Everything else is equal. We will call this algorithm "MAX." The second reference algorithm that we use is the "RANDOM" algorithm, which simply consists in random action selection (and so is not an active learning algorithm, but serves as a baseline).

IAC, MAX, and RANDOM will be compared in terms of their performance in generalization in predicting the consequence of actions during which a sound within the $f_{2}$ zone is emitted. This means that we will evaluate each of them in the part which we know is interesting. However, the whole space with all ranges of frequencies is made available to the robot, which does not know initially that there is a particular zone where it can actually learn nontrivial things.

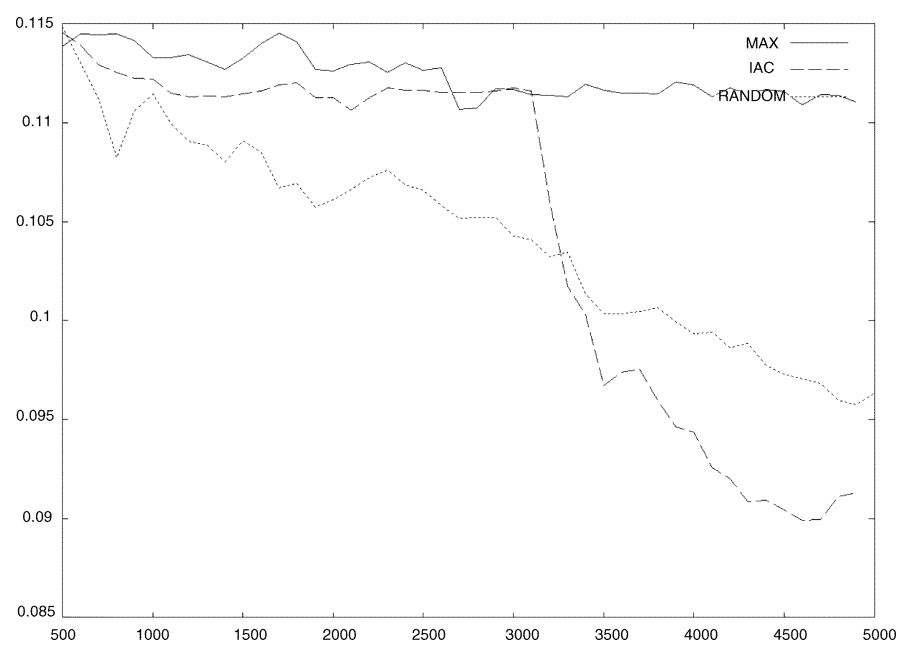

Fig. 6. Evolution of the performance in generalization (mean-squared prediction error) in situations in which the frequency of the emitted sound is within $f_{2}$ and, respectively, for the $M A X$ algorithm (continuous line), the IAC algorithm (long dashes line), and the RANDOM algorithm (small dashes lines). This allows us to compare how much the robot has learned of the interesting situations after a given number of performed actions, when it uses a given action selection algorithm.

For a given simulation using a given algorithm among IAC, MAX, and RANDOM, we evaluate every 100 actions of performance in generalization of the current learning machine. To do this, we initially made a simulation with random action selection and collected a database of input-output by storing the experienced $(\mathbf{S M}(\mathbf{t}), \mathbf{S}(\mathbf{t}+\mathbf{1}))$ couples for which the action included an emission of a sound with a frequency within $f_{2}$. This provides an independent test set which we used to test the capacity of prediction that the robot acquired at a given time in its development. For this test which is done every 100 actions, we freeze the learning machine and make it predict the output corresponding to all the inputs in the test database. The freezing ensures that the machine does not learn while it is tested. The prediction accuracy is measured using the mean-squared error over the database. After evaluating the performance, we unfreezed the system until the next evaluation.

Fig. 6 shows typical resulting curves of the three algorithms. We see that initially, the algorithm which learns fastest is the RANDOM algorithm. This is normal since MAX spends times in uninteresting situations, and IAC at the beginning spends time in the easy situation, so RANDOM is the algorithm which provides initially the highest amount of examples related to the production of the sounds with frequencies within $f_{2}$ (33\% of examples are of this type in this case). Then, after 3000 actions, the curve corresponding to the IAC algorithm suddenly drops down, this corresponds to the shift of attention of the robot towards situations with sounds with frequencies within $f_{2}$. Now, this robot spends $85 \%$ of its time in situations with sound with frequency within $f_{2}$ (and not $100 \%$ due to the 0.15 probability to do a random action). Quickly, the curve gets significantly below the RANDOM algorithm, and reaches a low plateau around 5000 actions (where the mean prediction error stays around 0.09). The RANDOM curve reaches a low plateau much later (this is not represented on this curve) after about 11000 actions. The value of the plateau, interestingly, is higher 
than with the IAC algorithm, it is 0.096 . We repeated the experiments 100 times in order to see whether this had some statistical significance. In each simulation, we measured the time where a plateau was reached (defined as 500 successive points, where the mean-squared error has a variance smaller than 0.0001), and what the mean-squared error was at that time. It turned out that the plateau was reached at $t=4583$ in average for IAC, with a standard deviation of 452, and at $t=11980$ in average with a standard deviation of 561 for RANDOM. The mean-squared error was $e=0.89$ in average with a standard deviation of 0.009 for IAC, and was $e=0.96$ with a standard deviation of 0.004 for RANDOM. As a consequence, we can say consistently that IAC allows the robot to learn the interesting part of the mapping about 2.6 times faster and with a higher performance in generalization than the RANDOM algorithm. This increase of the performances in generalization is similar to what has already been described in other active learning algorithms [32].

\section{F. Summary}

With this experiment, we have shown a first embodiment of the IAC system within a simulated robot. This has allowed us to show how IAC could manage the development of the robot in an inhomogeneous sensorimotor environment with parts which were not learnable by the robot. We have shown how the robot consistently avoided this zone of unlearnability and, on the other hand, explored autonomously sensorimotor situations of increasing complexity. This simple setup also allowed us to detail the evolution of the internal structures built by the IAC system. We could explain, for example, the progressive formation of regions with varying potentials for learning progress. Finally, this setup not only allowed us to show the interest of IAC as an intrinsic motivation system which could self-organize the behavior of a robot in a developmental manner, but it also showed that IAC is an efficient and robust active learning system. Indeed, we proved that it was faster than both the RANDOM algorithm and traditional active learning methods which are not suited to mappings with strong inhomogeneities and even unlearnable parts.

However, the simplicity of this setup did not allow us to show how a developmental sequence with more than one transition could self-organize autonomously (here, there was only a transition between a stage in which the robot focused on actions with sounds in $f_{1}$, and then a stage in which the robot focused on actions with sounds in $f_{2}$ ). We are now going to present a more complex experiment in which we will show that multiple sequential levels of self-organization of the behavior of the robot can happen.

\section{The Playground EXPERIMENT: THE Discovery OF SENSORIMOTOR AFFORDANCES}

This new experimental setup is called "The Playground Experiment." This involves a physical robot as well as a more complex sensorimotor system and environment. We use a Sony AIBO robot which is put on a baby play mat with various toys that can be bitten, bashed, or simply visually detected (see Fig. 7). The environment is very similar to the ones in

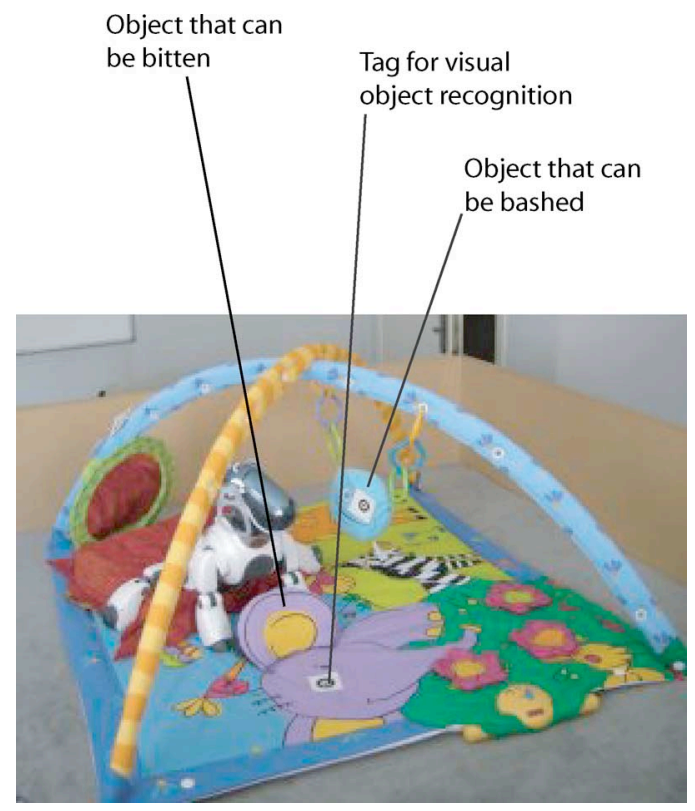

Fig. 7. The playground experiment setup.

which two- or three-month old children learn their first sensorimotor skills, although the sensorimotor apparatus of the robot here is much more limited. We have developed a web site which presents pictures and videos of this setup: http://playground.csl.sony.fr/.

\section{A. Motor Control}

The robot is equipped initially only with simple motor primitives. In particular, it is not able to walk around. There are three basic motor primitives: turning the head, bashing, and crouch biting. Each of them is controlled by a number of real number parameters, which are the action parameters that the robot controls. The "turning head" primitive is controlled with the pan and tilt parameters of the robot's head. The "bashing" primitive is controlled with the strength and the angle of the leg movement (a lower level automatic mechanism takes care of setting the individual motors controlling the leg). The "crouch biting" primitive is controlled by the depth of crouching (and the robot crouches in the direction in which it is looking at, which is determined by the pan and tilt parameters). To summarize, choosing an action consists in setting the parameters of the five-dimensional continuous vector $\mathbf{M}(\mathbf{t})$

$$
\mathbf{M}(\mathbf{t})=\left(p, t, b_{s}, b_{a}, d\right)
$$

where $p$ is the pan of the head, $t$ the tilt of the head, $b_{s}$ the strength of the bashing primitive, $b_{a}$ the angle of the bashing primitive, and $d$ the depth of the crouching of the robot for the biting motor primitive. All values are real numbers between 0 and 1 , plus the value -1 which is a convention used for not using a motor primitive, for example, $\mathbf{M}(\mathbf{t})=(0.3,0.95,-1,-1,0.29)$ corresponds to the combination of turning the head with parameters $p=0.3$ and $t=0.95$ with the biting primitive with the parameter $d=0.29$ but with no bashing movement. 


\section{B. Perception}

The robot is equipped with three high-level sensors based on lower level sensors. The sensory vector $\mathbf{S}(\mathbf{t})$ is thus three-dimensional

$$
\mathbf{S}(\mathbf{t})=\left(O_{v}, B_{i}, O_{s}\right)
$$

where:

- $O_{v}$ is the binary value of an object visual detection sensor: It takes the value 1 when the robot sees an object, and 0 in the other case. In the playground, we use simple visual tags that we stick on the toys and are easy to detect from the image processing point of view. These tags are black and white patterns similar to the Cybercode system developed by Rekimoto ([43]).

- $B_{i}$ is the binary value of a biting sensor: It takes the value 1 when the robot has something in its mouth and 0 otherwise. We use the cheek sensor of the AIBO.

- $O_{s}$ is the binary value of an oscillation sensor: It takes the value 1 when the robot detects that there is something oscillating in front of it, and 0 , otherwise. We use the infrared distance sensor of the AIBO to implement this high-level sensor. This sensor can detect, for example, when there is an object that has been bashed in the direction of the robot's gaze, but can also detect events due to human walking around the playground (we do not control the environment).

It is crucial to note that initially the robot knows nothing about sensorimotor affordances. For example, it does not know that the values of the object visual detection sensor are correlated with the values of its pan and tilt. It does not know that the values of the biting or object oscillation sensors can become 1 only when biting or bashing actions are performed towards an object. It does not know that some objects are more prone to provoke changes in the values of the $B_{i}$ and $O_{s}$ sensors when only certain kinds of actions are performed in their direction. It does not know, for example, that to get a change in the value of the oscillation sensor, bashing in the correct direction is not enough, because it also needs to look in the right direction (since its oscillation sensors are on the front of its head). These remarks allow us to understand easily that a random strategy will not be efficient in this environment. If the robot would do random action selection, in a vast majority of cases, nothing would happen (especially for the $B_{i}$ and $O_{s}$ sensors).

\section{The Action Perception Loop}

To summarize, the mapping that the robot has to learn is

$$
\begin{aligned}
f: \mathbf{S M}(\mathbf{t}) & =\left(p, t, b_{s}, b_{a}, d, O_{v}, B_{i}, O_{s}\right) \\
\longmapsto \mathbf{S}(\mathbf{t}+\mathbf{1}) & =\left(\widetilde{O_{v}}, \widetilde{B_{i}}, \widetilde{O_{s}}\right) .
\end{aligned}
$$

The robot is equipped with the IAC system, and thus chooses its actions according to the potential learning progress that it can provide to one of its experts. In this experiment, the action perception loop is rather long. When the robot chooses and executes an action, it waits until all its motor primitives have finished their execution, which lasts approximately one second, before choosing the next action. This is how the internal clock for the IAC system is implemented. On the one hand, this allows the robot to make all the measures necessary for determining adequate values of $\left(O_{v}, B_{i}, O_{s}\right)$. On the other hand, and most importantly, this allows the environment to come back to its "resting state." This means that the environment has no memory: after an action has been executed by the robot, all the objects are back in the same state. For example, if the object that can be bashed has actually been bashed, then it has stopped oscillating before the robots tries a new action. This is a deliberate choice to have an environment with no memory, while keeping all the advantages, the constraints and the complexity of a physical embodiment, this makes the mapping from actions to perception learnable in a reasonable time. This is crucial if one wants to do several experiments (already in this case, each experiment lasts for nearly one day). Furthermore, introducing an environment with memory frames the problem of the maximization of internal reward within delayed reward reinforcement problems, for which there exists powerful but complicated techniques whose biases would certainly make the results more complex and render them more difficult to interpret.

\section{Results}

During an experiment, we continuously measure a number of features which help us characterize the dynamics of the robot's development. First, we measure the frequency of the different kinds of actions that the robot performs in a given time window. More precisely:

- the percentage of actions which do not involve the biting and the bashing motor primitive in the last 100 actions (i.e., the robot's action boils down to "just looking" in a given direction);

- the percentage of actions which involve the biting motor primitive in the last 100 actions;

- the percentage of action which involve the bashing motor primitive in the last 100 action.

Then, we track the gaze of the robot and at each action, measure if it is looking towards: 1) the bitable object, or 2) the bashable object, or 3) no object. This is possible from an external point of view since we know where the objects are and so it is easy to derive the information from the head position.

Third, we measure the evolution of the frequency of successful biting actions and the evolution of successful bashing actions. A successful biting action is defined as an action which provokes a " 1 " value on the $B_{i}$ sensor (an object has actually be bitten). A successful bashing action is defined as an action which provokes an oscillation in the $O_{s}$ sensor.

Fig. 8 shows an example of the results of the evolution of the three kinds of measures on three different levels. A striking feature of these curves is the formation of sequences of peaks. Each of these peaks basically means that at the moment it occurs the robot is focusing its activity and its attention on a small subset of the sensorimotor space. Therefore, it is qualitatively different from random action performance in which the curves would be stationary and rather flat. By looking in detail at these peaks and at their co-occurence (or not) within the different kinds of measures, we can make a description of the evolution of the robot's behavior. In Fig. 8, we have marked a number of such peaks with letters from A to G. We can see that before the first peak, 


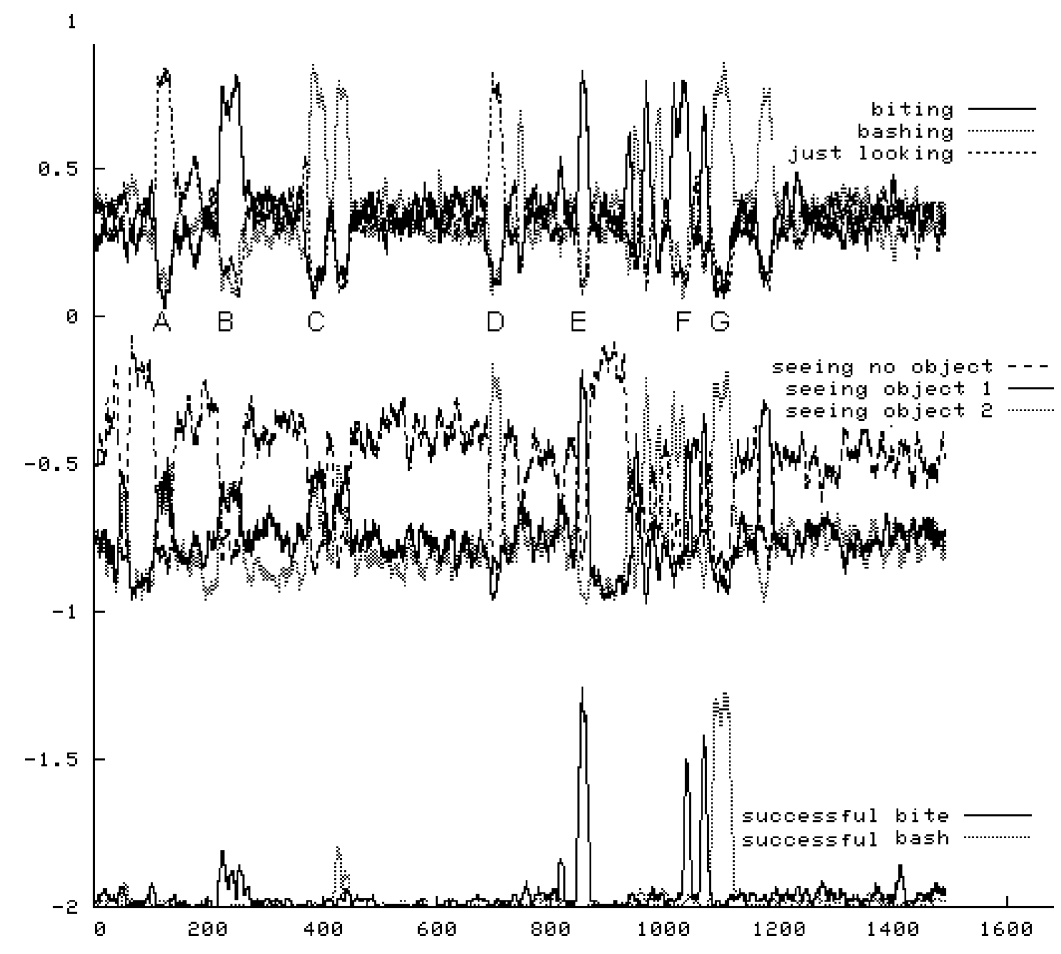

Fig. 8. Curves describing a run of the playground experiment. Top 3: Frequencies for certain action types on windows 100 time steps wide. Mid 3: Frequencies of gaze direction towards certain objects in windows 200 time steps wide: "object 1" refers to the bitable object, and "object 2" refers to the bashable object. Bottom 3: Frequencies of successful bite and successful bash in windows 200 time steps wide.

there is an initial phase during which all actions are produced equally often, that most often no object is seen, and that a successful bite or bash only happens extremely rarely. This corresponds to a phase of random action selection. Indeed, the robot initially categorizes the sensorimotor space using only one big region (and so there is only one category), and so all actions in any contexts are equally interesting. Then, we observe a peak (A) in the "just looking" curve. This means that for a while, the robot stops biting and bashing, and focuses on just moving its head around. This means that at this point the robot has split the space into several regions, and that a region corresponding to the sensorimotor loop of "just looking around" is associated to the highest learning progress from the robot's point of view. Then, the next peak (B) corresponds to a focus on the biting action primitive (with various continuous parameters), but it does not co-occur with looking towards the bitable object. This means that the robot is basically trying to bite in all directions around it, it did not discover yet the affordances of the biting actions with particular objects. The next peak (C) corresponds to a focus on the bashing action primitive (with various continuous parameters), but again the robot does not look towards a particular direction. As the only way to discover that a bashing action can make an object move is by looking in the direction of this object (because the IR sensor is on the cheek), this means that the robot does not use at this point the bashing primitive with the right affordances. The next peak (D) corresponds to a period within which the robot again stops biting and bashing and concentrates on moving the head, but this time we observe that the robot focuses these "looking" movements in a narrow part of the visual field, it is basically looking around one of the objects, learning how it disappears/reappears in its field of view.
Then, there is peak (E) corresponding to focusing on the biting action, which is this time coupled with a peak in the curve monitoring the looking direction towards the bitable object, and a peak in the curve monitoring the success in biting. It means that during this period, the robot uses the action primitive with the right affordances, and manages to bite the bitable object quite often. This peak is then repeated a little bit later (F). Finally, a co-occurrence of peaks $(\mathrm{G})$ appears that corresponds to a period during which the robot concentrates on using the bashing primitive with the right affordances, managing to actually bash the bashable object quite often.

This example shows that several interesting phenomena have appeared in this run of the experiment. First of all, the presence and co-occurrence of peaks of various kinds shows a self-organization of the behavior of the robot, which focuses on particular sensorimotor loops at different periods in time. Second, when we observe these peaks, we see that they are not random peaks, but show a progressive increase in the complexity of the behavior to which they correspond. Indeed, one has to remember that the intrinsic dimensionality of the "just looking" behavior (pan and tilt) is lower than the one of the "biting" behavior (which adds the depth of the crouching movement), which is in itself lower than the one of the "bashing" behavior (which adds the angle and the strength dimensions). The order of appearance of the periods within which the robot focuses on one of these activities is precisely the same. If we look in more detail, we also see that the biting behavior appears first in a nonaffordant version (the robot tries to bite things which cannot be bitten), and then only later in the affordant version (where it tries to bite the biteable object). The same observation holds for the bashing behavior: first, it appears without the right affordances, and then 
it appears with the right affordances. The formation of focused activities whose properties evolve and are refined with time can be used to describe the developmental trajectories that are generated in terms of stages. Indeed, one can define that a new stage begins when a co-occurence of peaks that never occurred before happens (and which denotes a novel kind of focused activity).

We ran the experiment several times with the real robots, and whereas each particular experiment produced curves which were different in the details, it seemed that some regularities in the patterns of peak formation, and in terms of stage sequences were present. We then proceeded to more experiments in order to precisely assess the statistical properties of these self-organized developmental trajectories. As each experiment with the real robot lasts several hours, and in order to be able to run many experiments (200), we developed a model of the experimental setup. Thanks to the fact that the physical environment was memoryless after each action of the robot, it was possible to make an accurate model of it using the following procedure. We let the robot perform several thousand actions and we recorded each time $S M(t)$ and $S(t+1)$. Then, from this database of examples, we trained a prediction machine based on locally weighted regression [44]. This machine was then used as a model of the physical environment and the IAC algorithm of the robot was directly plugged into it.

Using this simulated world setup, we ran 200 experiments, each time monitoring the evolution using the same measures as above. We then constructed higher level measures on each of the runs, based on the structure of the peak sequence. Peaks were defined here using a threshold on the height and width of the bumps in the curves. These measures correspond to the answers to these following questions.

- (Measure 1) Number of peaks?: How many peaks are there in the action curves (top curves)?

- (Measure 2) Complete scenario?: Is the following developmental scenario matched: first, there is a "just looking" peak, then there is a peak corresponding to "biting" with the wrong affordances which appears before a peak corresponding to "biting" with the right affordances, and there is a peak corresponding to "bashing" with the wrong affordances which appears before a peak corresponding to "bashing" with the right affordances (and the relative order between "biting"-related peaks and "bashing"-related peaks is ignored). Biting with the right affordance is defined here as the co-occurence between a peak in the "biting" curve and a peak in the "seeing the biteable object" curve, and biting with the wrong affordances is defined as all other situations. The corresponding definition applies to "bashing."

- (Measure 3) Nearly complete scenario?: Is the following less constrained developmental scenario matched. There is a peak corresponding to "biting" with the wrong affordances which appears before a peak corresponding to "biting" with the right affordances, and there is a peak corresponding to "bashing" with the wrong affordances which appears before a peak corresponding to "bashing" with the right affordances (and the relative order between "biting"-related peaks and "bashing"-related peaks is ignored).
TABLE I

Statistical Measures ON THE 200 SimUlation-BASED ExPERIMENTS

\begin{tabular}{|l|l|}
\hline Measures & Results \\
\hline (1) Number of peaks? & 9.67 \\
\hline (2) Complete scenario? & Yes: $34 \%$, No: $\mathbf{6 6} \%$ \\
\hline (3) Near complete scenario? & Yes: $\mathbf{5 3} \%$, No: $47 \%$ \\
\hline (4) Non-affordant bite before affordant bite? & Yes: $\mathbf{9 3} \%$, No: $7 \%$ \\
\hline (5) Non-affordant bash before affordant bash? & Yes: $\mathbf{5 7} \%$, No: $43 \%$ \\
\hline (6) Period of systematic successful bite? & Yes: $\mathbf{1 0 0} \%$, No: $0 \%$ \\
\hline (7) Period of systematic successful bash? & Yes: $\mathbf{7 8} \%$, No: $11 \%$ \\
\hline (8) Bite before bash? & Yes: $\mathbf{9 2} \%$, No: $8 \%$ \\
\hline (9) Successful bite before successful bash? & Yes: $\mathbf{7 7} \%$, No: $23 \%$ \\
\hline
\end{tabular}

- (Measure 4) Nonaffordant bite before affordant bite?: Is there is a peak corresponding to "biting" with the wrong affordances which appears before a peak corresponding to "biting" with the right affordances?

- (Measure 5) Nonaffordant bash before affordant bash?: Is there a peak corresponding to "bashing" with the wrong affordances which appears before a peak corresponding to "bashing" with the right affordances?

- (Measure 6) Period of systematic successful bite?: Does the robot succeed systematically in biting often at some point (= is there a peak in the "successful bite" curve)?

- (Measure 7) Period of systematic successful bash?: Does the robot succeed systematically in bashing often at some point (= is there a peak in the "successful bash" curve?

- (Measure 8) Bite before bash?: Is there a focus on biting which appears before a focus on bashing (independantly of affordance)?

- (Measure 9) Successful bite before successful bash?: Is there a focus on successfully biting which appear before a focus on successfully bashing?

The numerical results of these measures are summarized in Table I. This table shows that indeed some structural and statistical regularities arise in the self-organized developmental trajectories. First of all, one has to note that the complex and structured trajectory described by Measure 2 appears in $34 \%$ of the cases, which is high given the number of possible co-occurences of peaks which define a combinatorics of various trajectories. Furthermore, if we remove the test on "just looking," we see that in the majority of experiments, there is a systematic sequencing from nonaffordant to affordant actions for both biting and bashing. This shows an organized and progressive increase in the complexity of the behavior. Another measure confirms this increase of complexity from another point of view: if we compare the relative order of appearance of periods of focused bite or bash, then we find that "focused bite" appears in the large majority of the cases before the "focused bash," which corresponds to their relative intrinsic dimension ( 3 for biting and 4 for bashing). Finally, one can note that the robot in $100 \%$ of the experiments, reaches a period during which it repeatedly manages to bite the biteable object, and in $78 \%$ of the experiments, 


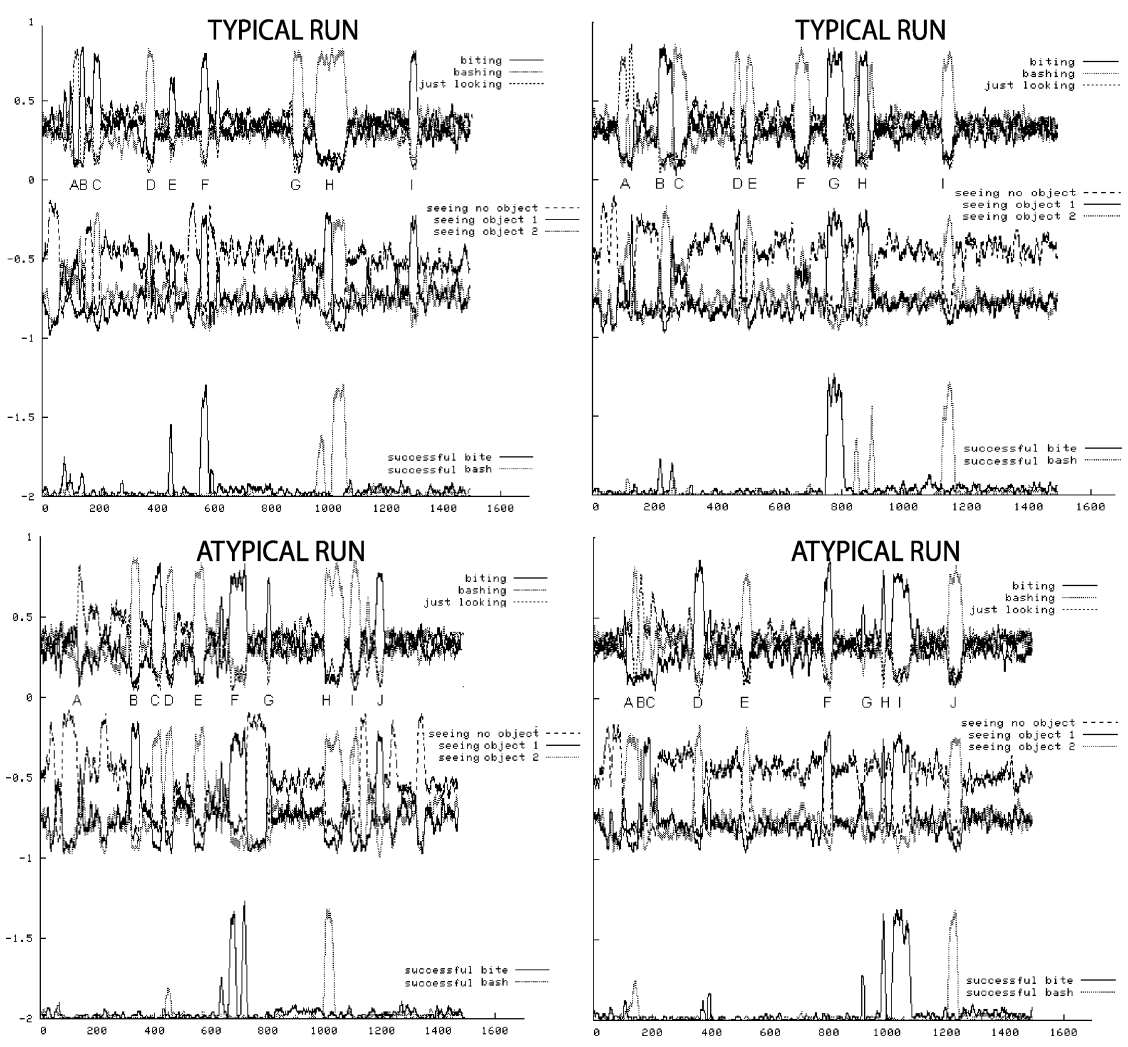

Fig. 9. Various runs of the simulated experiments. In the top squares, we observe two typical developmental trajectories corresponding to the "complete scenario" described by Measure 1. In the bottom curve, we observe rare but existing developmental trajectories.

it reaches a period during which it repeatedly manages to bash the bashable object. This last point is interesting since the robot was not preprogrammed to achieve this particular task.

These experiments show how the intrinsic motivation system which is implemented (IAC) drives the robot into a self-organized developmental trajectory in which periods of focused sensorimotor activities of progressively increasing complexity arise. We have seen that a number of structural regularities arose in the system, such as the tendancy of nonaffordant behavior to be explored before affordant behavior, or the tendancy to explore a certain kind of behavior (bite) before another kind (bash). Yet, one has also to stress that these regularities are only statistical: two developmental trajectories are never exactly the same, and more importantly, it happens that some particular trajectories observed in some experiments differ qualitatively from the mean. Fig. 9 illustrates this point. The figures on the top-left and top-right corners present runs which are very typical and correspond to the "complete scenario" described by Measure 1. On the contrary, the runs presented on the bottom-left and bottom-right corners correspond to atypical results. The experiment of which curves are presented in the bottom-left corner shows a case where the focused exploration of bashing was performed before the focused exploration of biting. Nevertheless, in this case, the regularity "nonaffordant before affordant" is preserved. On the bottom-right corner, we observe a run in which the affordant bashing activity appears very early and before any other focused activity. This balance between statistical regularities and diversity has parallels in infant sensorimotor development [45]. There are some strong structural regularities but from individual to individual there can be some substantial differences (e.g., some infants learn how to crawl before they can sit and others do the reverse).

\section{DISCUSSION}

\section{A. Developing Complex Behavioral Schemas}

We have discussed how to design a system of internal rewards suited for active and autonomous development. Such an intrinsic motivation system permits us to realize an efficient active exploration of a given sensorimotor space. In the experiments described, we deliberately considered simple spaces. Enhancing the complexity of perception and motor spaces seems crucial in order to expect the emergence of more complex forms of behavior. However, designing suitable spaces that can lead to complex behavioral patterns raises several difficult issues.

A first issue is whether perception and motor spaces should be considered as two independent spaces. The intrinsic links that bind perception with action have been stressed by many authors. In some circumstances, relevant information about a given environment arises from sensorimotor trajectories rather than from simple analysis of perceptual data. Several experiments have shown that agents can simplify problems of categorizing situations by actively modifying their own position or orientation with respect to the environment or by modifying the environment itself. In the same manner, certain environmental regularities can be detected only by producing a particular stereotyped behavior (e.g., [46] and [47]). The fact that perception is fundamentally active, naturally leads to consider 
abstractions like behavioral schemas as relevant unit for understanding development.

Schemas are famously known as central elements of Piaget's developmental psychology but the term has also been used in neurology, cognitive psychology, and motor control [48, pp. 36-40], and related notions appeared in artificial intelligence under names like frames or scripts [49], [50]. In Piaget's theory, children's development can be interpreted as the incremental organization of a set of schemas. Schemas are skills that serve both the perceiving of the environment and acting upon it. Piaget calls assimilation the ability to make sense of a situation in terms of a current set of schemas, and accommodation the way in which schemas are updated as the expectations based on assimilation are not met. The child starts with basic sensorimotor schemas such as suckling, grasping, and some primary forms of eye-hand coordination. Through accommodation and assimilation, new schemas are created, and sets of existing schemas get coordinated. The child makes progressively more complex abstract inferences about the environment, leading eventually to language and logic, forms of abstract thought that are no longer directly grounded, in particular, sensorimotor situations. The whole developmental trajectory can be interpreted as an extension from a simple sensorimotor space to an elaborated mental space. The space changes but the fundamental dynamics of accommodation and assimilation that actively drive the child's behavior remain the same.

It is important to stress that schemas are primarily functional units. In that sense, they are a priori distinct from structural units that can be identified in the organization of the organism or the machine that produces the observed behavior. However, many artificial intelligence models make use of internal explicit schema structures. In such systems, there is a one-to-one mapping between these internal structures and the functional operation that the agent can perform. For instance, Drescher describes a system inspired by Piaget's theories in which a developing agent explicitly creates, modifies, and merges schema structures in order to interact with a simple simulated environment [51]. Using explicit schema structures has several advantages: such structures can be manipulated via symbolic operations, creation of new skills can be easily monitored by following the creation of new schemas, etc.

Other systems do not rely on such explicit representations. These are typically subsymbolic systems, using continuous representations of their environment. Nevertheless, such systems may display some organized forms of behavior where clear functional units can be identified. Their developmental trajectories can also be interpreted as a progressive organization of schemas. For instance, the developmental trajectories produced by the typical experiments of Section VII can be interpreted as assimilation and accommodation phases. In these typical runs, the robot "discovers" the biting and bashing schema by producing repeated sequences of these kinds of behavior, but initially these actions are not systematically oriented towards the biteable or the bashable object. This stage corresponds to "assimilation." It is only later that "accommodation" occurs as biting and bashing starts to be associated with their respective appropriate context of use. Our experiments show that functional organization can emerge even in the absence of explicit internal schema structures. However, the current limitations of such a system may appear when considering more complex forms of behavioral organization such as formation of hierarchical structures and the emergence of goals.

1) Hierarchical Organization: Complex behavior patterns are hierarchically organized. For instance, a complex motor program is often described as an abstract event sequence at a high level and a detailed motor program in a lower level. Therefore, possibility for forming level structures is a key issue. Different authors have already tried to tackle how combinations of primitives could be autonomously organized in higher level structures. Option theory offers an interesting mathematical framework to address hierarchical organization of systems using explicit schema structures [52]. Options are like subroutines associated with closed-loop control structures. They can invoke other options as components. Barto et al. have recently illustrated in a simple environment how options could be used to develop a hierarchical collection of skills [21]. Hierarchical organization of explicit schemas is also illustrated by the work of Drescher among others [51]. However, can hierarchically organized behavior appear in the absence of explicit schemas? Different attempts have been made in this direction. A multiple model-based reinforcement learning capable of decomposing a task based on predictability levels was proposed by Doya et al. [53]. Tani and Nolfi presented a system capable of combining local experts using gated modules [54]. However, in all these studies, explicit level structures are predetermined by the network architecture. The question of whether hierarchical structures can simply self-organize without being explicitly programmed remains open.

2) Goal-Directedness: Complex behavior patterns are also associated with intentionally directed processes. This means that they are performed by an agent trying to achieve a particular desirable situation that constitutes its aim or goal (e.g., reducing hunger, following someone, learning something). The agent's behavior reflects his or her intention, that is the plan of action that the agent chooses for realizing this particular goal. This plan includes both the means and the pursued goal [55]. Once again, systems using explicit schema structure embed these notions of goals and means as explicit symbolic representations. Such explicit goals can be created, updated, deleted, and more importantly, easily monitored. This has led to numerous systems in classical artificial intelligence, and research in this area has influenced the importance of the way we consider decision making or planning. More recently, research on agent architectures [56] has put a major emphasis on the same issues. However, these models do not give much insight on the developmental and cognitive mechanisms that lead to the notion of intentionally directed behavior. Can goals and means simply emerge out of subsymbolic dynamics? This is one of the most challenging issues that developmental approaches to cognition have to face [57]. To some extent, certain reinforcement learning models have demonstrated that the organization of behavior into goals and subgoals can be interpreted as emergent features resulting in simpler drives [37]. However, no subsymbolic systems currently matches the performances and the flexibility of systems using explicit goal-directed schemas. 
3) Generalization, Transfer, Analogy: Generalization, transfer or analogies between schemas are also thought to be central for the emergence of complex behavior patterns (see [58] for a general discussion of the issue of transfer in cognition). Skills do not develop independently from one another. The ones that have structural relationship bootstrap each other. In particular, processes of analogy and metaphors are crucial for transferring know-how developed in sensorimotor contexts to more abstract spaces [59]. There is an important literature on how to compare explicit schema structure (e.g., [60]), but many authors have argued that generalization and transfer of skills could also be (maybe even more) efficient in the absence of symbolic representation [61]. This debate bears some resemblance with the opposition between localists or distributed kinds of representation. Systems with explicit schema structures, but also many subsymbolic systems using memories organized into local structures (e.g., sets of experts) are called localists. In this scheme, learning a new behavior schema corresponds to the addition of a template to an existing set of modules. The independence of the modules facilitates incremental learning as each addition does not cause interferences with the existing memory contents. However, extension to unknown patterns must be realized with ad hoc processes that specify the way similarity should be computed. In the same manner, generalization across a large set of local representations is intrinsically difficult. On the contrary, in systems with distributed representations, behavior schemas are not assigned to a particular module but are memorized in a distributed manner (e.g., as synaptic weights of global neural network). This means that each schema can only exist in relation to others. Self-organized generalization processes are facilitated in such context [62].

Developmental trajectories of intrinsically motivated agents are constrained by many factors. We have briefly discussed some of the important issues for designing systems capable of developing reusable, goal-directed, hierarchically organized behavioral schemas. Investigating the resulting dynamics of the intrinsic motivation systems embedded in such kinds of more complex spaces will be the topic of future research.

\section{B. Relation to Developmental Psychology}

Our research takes clear inspiration from developmental psychology both conceptually (the notion of intrinsic motivation originally comes from psychology) and methodologically (analysis of development in terms of qualitative sequences of different kinds of behavioral patterns). Could our model be interesting in return for interpreting processes underlying an infant's development? More precisely:

- Can we interpret a particular developmental process as being the result of a progress drive, an intrinsic motivation system driving the infant into situations expected to result in maximal learning progress?

- Can operant models of intrinsic motivation provide useful abstraction that address the complexity of infant's development?

Some initial attempts have been taken to start answering these questions. Taking ground on preliminary experimental results, we discussed in [63] a scenario presenting the putative role of the progress drive for the development of early imitation. We argue, in particular, that progress-driven learning could help understand why children focus on specific imitative activities at a certain age and how they progressively organize preferential interactions with particular entities present in their environment.

1) Progress Niches: To facilitate interpretation, we introduced the notion of progress niches to characterize the behavior of our model. The progress drive pushes the agent to discover and focus on situations which lead to maximal learning progress. These situations, neither too predictable nor too difficult to predict, are "progress niches." Progress niches are not intrinsic properties of the environment. They result from a relation between a particular environment, a particular embodiment (sensors, actuators, feature detectors, and techniques used by the prediction algorithms), and a particular time in the developmental history of the agent. Once discovered, progress niches progressively disappear as they become more predictable. The notion of progress niches is related to Vygotsky's zone of proximal development, where the adult deliberately challenges the child's level of understanding. Adults push children to engage in activities beyond their current mastery level, but not too far beyond so that they remain comprehensible [64]. We could interpret the zone of proximal development as a set of potential progress niches organized by the adult in order to help the child learn. However, it should be clear that independently of the adults' efforts, what is and what is not a progress niche is ultimately defined from the child's point of view. Progress niches also share similarities with Csikszentmihalyi's flow experiences [8]. Csikszentmihalyi argues that some activities are autotelic when challenges are appropriately balanced with the skills required to cope with them (see also [65]). We prefer to use the term progress niche by analogy with ecological niches as we refer to a transient state in the evolution of a complex "ecological" system involving the embodied agent and its environment.

2) Self-Other Distinction: Using this terminology, the computational model presented in this paper shows how an agent can: 1) separate its sensorimotor space into zones of different predictability levels and 2) choose to focus on the one which leads to maximal learning progress, called a "progress niche." With this kind of operant model, it could be speculated that meaningful sensorimotor distinctions (self, others, and objects in the environment) may be the result of discriminations constructed during a progress-driven process. We can more specifically offer an interpretation of several fundamental stages characterizing an infant's development during their first year.

- Stage 1: Like-me stance (0-1 m). Simple forms of imitative behavior have been argued to be present just after birth. They could constitute a process of early identification. Some totally or partially nativist explanations could account for this early "like-me stance" [66], [67]. This would suggest the possibility of an early distinction between persons and things. If an intermodal mapping facilitating the match between what is seen and what is felt exists, the hypothesis of a progress drive would suggest that infants will indeed create a discrimination between such easily predictable couplings (interaction with peers) and unpredictable situations (all the other cases) and that they will focus on the first zone of their sensorimotor space that 
constitutes a "progress niche." Neonates imitation (when it occurs) would be the result of the exploitation of the most predictable coupling present just after birth.

- Stage 2: Circular reactions (1-2 m). During the first two months of their life, infants perform repeated body motion. They kick their legs repeatedly, they wave their arms. This process is sometimes referred as "body babbling." However, nothing indicates that this exploratory behavior is randomly organized. Rochat argues that children are in fact performing self-imitation, trying to imitate themselves [68]. This would mean that children are structuring their own behavior in order to make it more predictable and form "circular reactions" this way [41], [69]. Such self-imitative behaviors can be well explained by the progress drive hypothesis. Sensorimotor trajectories directed towards the child's own body can be easily discriminated from trajectories directed towards other people by comparing their relative predictability. In many respects, making progress in understanding primary circular reactions is easier than in the cases involving other agents: Self-centered types of behavior are "progress niches." In such a scenario, the "self" emerges as a meaningful discrimination for achieving better predictability. Once this distinction is made, progress for predicting the effects of self-centered actions can be rapidly made.

- Stage 3: Self-other interactions $(2-4 \mathbf{~ m})$. After two months, infants become more attentive to the external world and particularly to people. Parental scaffolding plays a critical role for making the interaction with the child more predictable [70]. Parents adapt their own responses so that interactions with the child follow the normal social rules that characterize communicative exchanges (e.g., turn taking). Moreover, if an adult imitates an infant's own actions, it can trigger continued activity in the infant. This early imitative behavior is referred as "pseudo-imitation" by Piaget [71]. Pseudo-imitation and focusing on scaffolded adult behavior could be seen as predictable effects of the progress drive. As the self-centered trajectories start to become well mastered (and do not constitute "progress niches" anymore), the child's focus shifts to another branch of the discrimination tree, the "self-other" zone.

- Stage 4: Interactions with objects (5-7 m). After five months, attention shifts again from people to objects. Children gain increased control over the manipulation of some objects on which they discover "affordances" [72]. Parents recognize this shift and initiate interactions about those affordant objects. However, children do not easily alternate their attention between the object and their caregiver. A progress-driven process can account for this discrimination between affordant objects and unmastered aspects of the environment. Although this stage is typically not seen as imitative, it could be argued that the exploratory process involved in the discovery of the object affordances shares several common features with the one involved for selfcentered activities: the child structures its world looking for "progress niches."

We have to stress that the system discussed in this paper is not meant to reenact precisely the infant's developmental sequence, and it is not a model of human development. For instance, the playground experiment focuses directly on the discovery of object's affordances. However, in addition to the developmental robotics engineering techniques that it explores, we think that this system, as well as other existing intrinsic artificial motivation systems, can also be used as a "tool for thoughts" in developmental psychology. In that sense, it may help in formulating new concepts useful for the interpretation of the developmental dynamics underlying children's development. For example, the existence of a progress drive could explain why certain types of imitative behavior are produced by children at a certain age and stop being produced later on. It could also explain how discrimination between actions oriented towards the self, towards others, and towards the environment may occur. However, we do not even imagine that a drive for maximizing learning progress could be the only motivational principle driving children's development. The complete picture is likely to include a complex set of drives. Developmental dynamics are certainly the result of the interplay between intrinsic and extrinsic forms of motivations, in particular learning biases, as well as embodiment and environmental constraints. We believe that computational and robotic approaches can help specify the contribution of these different components in the overall observed patterns and shed new light on the particular role played by intrinsic motivation in these complex processes.

\section{CONCLUSION}

Intrinsic motivation systems are likely to play a pivotal role for the future of developmental robotics. In this paper, we have presented the background in developmental psychology, neuroscience, and machine learning. We showed that current efforts in the developmental robotics community are approaching the construction of intrinsic motivation systems through the operationalization and implementation of concepts such as "novelty," "surprise," or more generally "curiosity." We have reviewed some representative works in this direction, trying to classify them into different groups according to the way they operationalized curiosity. Then, we presented an intrinsic motivation system called IAC, which was conceived to drive the development of a robot in continuous noisy inhomogeneous environmental and sensorimotor spaces, permitting an autonomous self-organization of behavior into a developmental trajectory with sequences of increasingly complex behavioral patterns. This was made possible thanks to the way the system evaluates its own learning progress, through the combination of a regional evaluation of the similarity of situations with a smoothing of the error rate curves associated to each region.

This system was tested in two robotic setups. In a first simple simulated robotic setup, we showed in detail how the system works, and provokes both behavioral and cognitive development, by looking in detail into the traces of the simulation. This first setup also showed how IAC can allow a robot to avoid situations which are not learnable by the system, and engage in situations of progressively increasing complexity in terms of difficulty of learning, which leads to a self-organization of the behavior. This first setup finally allowed us to show that our intrinsic motivation system could be used efficiently as an active learning algorithm robust in inhomogeneous spaces. Some 
currently ongoing work suggests that these results still hold in high-dimensional continuous spaces. If this is confirmed, this would allow us to attack real-world learning problems whose properties of inhomogeneity kept them out of reach of standard active learning methods so far [33]. In a second real and more complex robotic setup, we showed how IAC can drive the development of a robot through more than one developmental transition, and thus allows the robot to autonomously generate a developmental sequence. Conducting these experiments also provided the opportunity to discuss methodological issues related to the evaluation of a developmental robot. Indeed, classical machine learning methods of evaluation, based on the measure of the performance of a system on a given human-defined task, are not suited for developmental robots since one of their key features is to be task-independent, as advocated by Weng [34]. We explained that a developmental evaluation should be based on the monitoring of the evolution of the complexity of the system from different points of view, since complexity is indeed a concept which is observer-dependent. For example, it is a necessity to couple a measure of the evolution of the complexity from the robot's point of view, and the monitoring of its behavior on a long time scale using methods inspired from human sciences and developmental psychology.

We have also discussed the limits of the system as we presented it in this paper. Indeed, there are two kinds of limitations which will be the subject of future work. On the one hand, we deliberately made the simplification that what the system should optimize is the immediate reward $(r(t+1))$. This allowed us not to use complex reinforcement techniques and limit the biases coming from the action selection procedure in order to better understand the properties of our learning progress measure. Nevertheless, this will be a necessity in the future to use such complex reinforcement learning techniques, since in the real-world progress niches are not always readily accessible, and thus comes the problems of delayed rewards. This extension of our system should certainly be inspired by the work of Barto et al. [21] who have presented a study which is very complementary to ours, in which they experimented the use of complex reinforcement techniques given a simple novelty-based intrinsic motivation system.

A second kind of limitation which characterizes the current system is the fact that the sensorimotor space is rather simple, in particular, from the point of view of representation. It is an open issue to study how forms of representations more complex than scalar vectors, such as schemas for example, could be integrated within the IAC system. One of the potential problems to be solved is if several levels of representations are used: How can one build measures of learning progress or knowledge gain which are homogeneous and allow the comparison of activities or sensorimotor contexts which involve different representations?

Finally, we have seen that even if the primary goal of the system we presented is to allow the construction of a truly developmental robot, taking inspiration from human development, the system could in return possibly be useful for developmental psychologists as a tool for thoughts. Indeed, we explained how it can help to formulate new concepts for the interpretation of the developmental dynamics involved in human infant's development.

\section{ACKNOWLEDGMENT}

The authors would like to thank A. Whyte whose help and programming skills were precious for conducting experiments permitting to test intrinsic motivation systems (in particular, he designed the motor primitives used by the robot in the Playground experiment), as well as J.-C. Baillie for letting us use the URBI system [73] for programming the robot and L. Steels for relevant comments on this work.

\section{REFERENCES}

[1] J. Weng, J. McClelland, A. Pentland, O. Sporns, I. Stockman, M. Sur, and E. Thelen, "Autonomous mental development by robots and animals," Science, vol. 291, pp. 599-600, 2001.

[2] M. Lungarella, G. Metta, R. Pfeifer, and G. Sandini, "Developmental robotics: A survey," Connection Sci., vol. 15, no. 4, pp. 151-190, 2003.

[3] M. Asada, S. Noda, S. Tawaratsumida, and K. Hosoda, "Purposive behavior acquisition on a real robot by vision-based reinforcement learning," Mach. Learn., vol. 23, pp. 279-303, 1996.

[4] J. Elman, "Learning and development in neural networks: The importance of starting small," Cognition, vol. 48, pp. 71-99, 1993.

[5] R. White, "Motivation reconsidered: The concept of competence," Psychol. Rev., vol. 66, pp. 297-333, 1959.

[6] E. Deci and R. Ryan, Intrinsic Motivation and Self-Determination in Human Behavior. New York: Plenum, 1985.

[7] D. Berlyne, Conflict, Arousal and Curiosity. New York: McGrawHill, 1960.

[8] M. Csikszenthmihalyi, Flow-the Psychology of Optimal Experience. New York: Harper Perennial, 1991.

[9] W. Schultz, P. Dayan, and P. Montague, "A neural substrate of prediction and reward," Science, vol. 275, pp. 1593-1599, 1997.

[10] P. Dayan and W. Belleine, "Reward, motivation and reinforcement learning," Neuron, vol. 36, pp. 285-298, 2002.

[11] S. Kakade and P. Dayan, "Dopamine: Generalization and bonuses," Neural Netw., vol. 15, pp. 549-559, 2002.

[12] J.-C. Horvitz, "Mesolimbocortical and nigrostriatal dopamine responses to salient non-reward events," Neuroscience, vol. 96, no. 4, pp. 651-656, 2000.

[13] M. Csikszentmihalyi, Creativity-Flow and the Psychology of Discovery and Invention. New York: Harper Perennial, 1996.

[14] J. Schmidhuber, "Curious model-building control systems," in Proc. Int. Joint Conf. Neural Netw., Singapore, 1991, vol. 2, pp. 1458-1463.

[15] S. Thrun, "Exploration in active learning," in Handbook of Brain Science and Neural Networks, M. Arbib, Ed. Cambridge, MA: MIT Press, 1995.

[16] J. Herrmann, K. Pawelzik, and T. Geisel, "Learning predicitve representations," Neurocomputing, vol. 32-33, pp. 785-791, 2000.

[17] J. Weng, "A theory for mentally developing robots," in Proc. 2nd Int. Conf. Development Learn., 2002, pp. 131-140.

[18] X. Huang and J. Weng, "Novelty and reinforcement learning in the value system of developmental robots," in Proc. 2nd Int. Workshop Epigenetic Robotics: Modeling Cognitive Development in Robotic Systems, C. Prince, Y. Demiris, Y. Marom, H. Kozima, and C. Balkenius, Eds., 2002, vol. 94, Lund University Cognitive Studies, pp. $47-55$.

[19] F. Kaplan and P.-Y. Oudeyer, "Motivational principles for visual know-how development," in Proc. 3rd Int. Workshop Epigenetic Robotics: Modeling Cognitive Development in Robotic Systems, C. Prince, L. Berthouze, H. Kozima, D. Bullock, G. Stojanov, and C. Balkenius, Eds., 2003, vol. 101, Lund University Cognitive Studies, pp. 73-80.

[20] J. Marshall, D. Blank, and L. Meeden, "An emergent framework for self-motivation in developmental robotics," in Proc. 3rd Int. Conf. Development Learn., San Diego, CA, 2004, pp. 104-111.

[21] A. Barto, S. Singh, and N. Chentanez, "Intrinsically motivated learning of hierarchical collections of skills," in Proc. 3rd Int. Conf. Development Learn., San Diego, CA, 2004, pp. 112-119.

[22] V. Fedorov, Theory of Optimal Experiment. New York, NY: Academic, 1972.

[23] D. Cohn, Z. Ghahramani, and M. Jordan, "Active learning with statistical models," J. Artif. Intell. Res., vol. 4, pp. 129-145, 1996.

[24] M. Hasenjager and H. Ritter, Active Learning in Neural Networks. Berlin, Germany: Physica-Verlag GmbH, 2002, Physica-Verlag Studies In Fuzziness and Soft Computing Series, pp. 137-169. 
[25] J. Denzler and C. Brown, "Information theoretic sensor data selection for active object recognition and state estimation," IEEE Trans. Pattern Anal. Mach. Intell., vol. 2, no. 24, pp. 145-157, Feb. 2002.

[26] M. Plutowsky and H. White, "Selecting concise training sets from clean data," IEEE Trans. Neural Netw., vol. 4, no. 2, pp. 305-318, Mar. 1993.

[27] T. Watkin and A. Rau, "Selecting examples for perceptrons," J. Physics A: Mathematical and General, vol. 25, pp. 113-121, 1992.

[28] D. MacKay, "Information-based objective functions for active data selection," Neural Comput., vol. 4, pp. 590-604, 1992.

[29] M. Belue, K. Bauer, and D. Ruck, "Selecting optimal experiments for multiple output multi-layer perceptrons," Neural Comput., vol. 9, pp. 161-183, 1997.

[30] G. Paas and J. Kindermann, "Bayesian query construction for neural network models," in Advances in Neural Processing Systems, G. Tesauro, D. Touretzky, and T. Leen, Eds. : MIT Press, 1995, vol. 7, pp. 443-450.

[31] K. O. M. Hasenjager and H. Ritter, Active Learning in Self-Organizing Maps. New York: Elsevier, 1999, pp. 57-70.

[32] D. Cohn, L. Atlas, and R. Ladner, "Improving generalization with active learning," Mach. Learn., vol. 15, no. 2, pp. 201-221, 1994.

[33] J. Poland and A. Zell, "Different criteria for active learning in neural networks: A comparative study," in Proc. 10th Eur. Symp. Artif. Neural Netw., M. Verleysen, Ed., 2002, pp. 119-124.

[34] J. Weng, "Developmental robotics: Theory and experiments," Int. J. Humanoid Robotics, vol. 1, no. 2, pp. 199-236, 2004.

[35] N. Roy and A. McCallum, "Towards optimal active learning through sampling estimation of error reduction," in Proc. 18th Int. Conf. Mach. Learn., 2001, pp. 441-448.

[36] R. Collobert and S. Bengio, "Svmtorch: Support vector machines for large-scale regression problems," J. Mach. Learn. Res., vol. 1, pp. 143-160, 2001.

[37] R. Sutton and A. Barto, Reinforcement Learning: An Introduction. Cambridge, MA.: MIT Press, 1998.

[38] C. Walkins and P. Dayan, "Q-learning," Mach. Learn., vol. 8, pp. 279-292, 1992.

[39] K. Kaneko and I. Tsuda, Complex Systems : Chaos and Beyond. Berlin, Germany: Springer-Verlag, 2000.

[40] O. Sporns and T. Pegors, "Information-theoretical aspects of embodied artificial intelligence," in Embodied Artificial Intelligence, F. Iida, R. Pfeifer, L. Steels, and Y. Kuniyoshi, Eds. Berlin, Germany: SpringerVerlag, 2003, LNAI 3139, pp. 74-85.

[41] J. Piaget, The Origins of Intelligence in Children. New York, NY: Norton, 1952.

[42] O. Michel, "Webots: Professional mobile robot simulation," Int. J. Advanced Robotic Syst., vol. 1, no. 1, pp. 39-42, 2004.

[43] J. Rekimoto and Y. Ayatsuka, "Cybercode: Designing augmented reality environments with visual tags," in Proc. Designing Augmented Reality Environments, 2000, pp. 1-10.

[44] S. Schaal, C. Atkeson, and S. Vijayakumar, "Scalable techniques from nonparameteric statistics for real-time robot learning," Appl. Intell., vol. 17, no. 1, pp. 49-60, 2002.

[45] E. Thelen and L. B. Smith, A Dynamic Systems Approach to the Development of Cognition and Action. Cambridge, MA: MIT Press, 1994.

[46] R. D. Beer, "The dynamics of active categorical perception in an evolved model agent," Adaptive Behav., vol. 11, no. 4, pp. 209-243, 2003.

[47] S. Nolfi and J. Tani, "Extracting regularities in space and time through a cascade of prediction networks," Connection Sci., vol. 11, no. 2, pp. 129-152, 1999.

[48] M. Arbib, The Handbook of Brain Theory and Neural Networks. Cambridge, MA: MIT Press, 2003.

[49] M. Minsky, "A framework for representing knowledge," in The Psychology of Computer Vision, P. Wiston, Ed. New York: McGrawHill, 1975, pp. 211-277.

[50] R. Schank and R. Abelson, Scripts, Plans, Goals and Understanding: An Inquiry into Human Knowledge Structures. Hillsdale, NJ.: Lawrence Erlbaum, 1977.

[51] G. L. Drescher, Made-Up Minds. Cambridge, MA.: MIT Press, 1991.

[52] R. Sutton, D. Precup, and S. Singh, "Between MDPSs and semi-MDPS: A framework for temporal abstraction in reinforcement learning," Artif. Intell., vol. 112, pp. 181-211, 1999.

[53] K. Doya, K. Samejima, K. Katagiri, and M. Kawato, "Multiple model-based reinforcement learning," Neural Comput., vol. 14, pp. 1347-1369, 2002.
[54] J. Tani and S. Nolfi, "Learning to perceive the world as articulated: An approach for hierarchical learning in sensory-motor systems," Neural Netw., vol. 12, pp. 1131-1141, 1999.

[55] M. Tomasello, M. Carpenter, J. Call, T. Behne, and H. Moll, "Understanding and sharing intentions: The origins of cultural cognition," Behav. Brain Sci., vol. 28, no. 5, pp. 675-691, 2005.

[56] F. Dignum and R. Conte, "Intentional agents and goal formation," in Proc. 4th Int. Workshop Intell. Agents IV, Agent Theories, Architectures, and Languages, London, U.K., 1997, vol. 1365, LNCS, pp. 231-243.

[57] F. Kaplan and V. Hafner, "The challenges of joint attention," Interaction Studies, vol. 7, no. 2, pp. 128-134, 2006.

[58] A. Robins, "Transfer in cognition," Connection Sci., vol. 8, no. 2, pp. 185-204, 1996.

[59] G. Lakoff and M. Johnson, Philosophy in the Flesh: The Embodied Mind and its Challenge to Western Thought. New York: Basic Books, 1998.

[60] D. Gentner, K. Holyoak, and N. Kokinov, The Analogical Mind: Perspectives from Cognitive Science. Cambridge, MA: MIT Press, 2001.

[61] L. Pratt and B. Jennings, "A survey of connectionist network reuse through transfer," Connection Sci., vol. 8, no. 2, pp. 163-184, 1996.

[62] J. Tani, M. Ito, and Y. Sugita, "Self-organization of distributedly represented multiple behavior schema in a mirror system," Neural Netw., vol. 17, pp. 1273-1289, 2004.

[63] F. Kaplan and P.-Y. Oudeyer, "The progress-drive hypothesis: An interpretation of early imitation," in Models and Mechanisms of Imitation and Social Learning: Behavioral, Social and Communication Dimensions, K. Dautenhahn and C. Nehaniv, Eds. Cambridge, U.K.: Cambridge Univ. Press, 2007, pp. 361-377.

[64] L. Vygotsky, Mind in Society. Cambridge, MA: Harvard Univ. Press, 1978, The Development of Higher Psychological Processes.

[65] L. Steels, "The autotelic principle," in Embodied Artificial Intelligence, I. Fumiya, R. Pfeifer, L. Steels, and K. Kunyoshi, Eds. Berlin, Germany: Springer-Verlag, 2004, vol. 3139, Lecture Notes in AI, pp. 231-242.

[66] A. Meltzoff and A. Gopnick, "The role of imitation in understanding persons and developing a theory of mind," in Understanding Other Minds, H. T.-F. S. Baron-Cohen and D. Cohen, Eds. Oxford, U.K.: Oxford Univ. Press, 1993, pp. 335-366.

[67] C. Moore and V. Corkum, "Social understanding at the end of the first year of life," Developmental Rev., vol. 14, pp. 349-372, 1994.

[68] P. Rochat, "Ego function of early imitation," in The Imitative Mind: Development, Evolution and Brain Bases, A. Melzoff and W. Prinz, Eds. Cambridge, U.K.: Cambridge Univ. Press, 2002.

[69] J. Baldwin, Mental Development in the Child and the Race. New York: Macmillan, 1925.

[70] H. Schaffer, "Early interactive development in studies of mother-infant interaction," in Proc. Loch Lomonds Symp., New York, 1977, pp. 3-18.

[71] J. Piaget, Play, Dreams and Imitation in Childhood. New York: Norton Press, 1962.

[72] J. Gibson, The Ecological Approach to Visual Perception. Mahwah, NJ: Lawrence Erlbaum, 1986.

[73] J.-C. Baillie, "Urbi: Towards a universal robotic low-level programming language," in Proc. IEEE Int. Conf. Intell. Robots Syst., Aug. 2005, pp. 820-825.

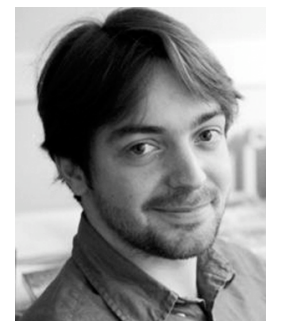

Pierre-Yves Oudeyer studied theoretical computer science at the Ecole Normale Supérieure de Lyon, Lyon, France, and received the Ph.D. degree in artificial intelligence from the University Paris VI, France.

$\mathrm{He}$ is a Researcher at the Sony Computer Science Laboratory since 1999. He has published a book, more than 50 papers in international journals and conferences, and received several prizes for his work in developmental robotics and on the origins of language. $\mathrm{He}$ is interested in the mechanisms that allow humans and robots to develop perceptual, motivational, behavioral, and social capabilities to become capable of sharing cultural representations. 


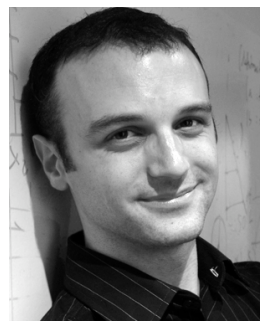

Frederic Kaplan graduated as an engineer of the Ecole Nationale Supérieur des Télécommunications, Paris, and received the Ph.D. degree in artificial intelligence from the University Paris VI.

$\mathrm{He}$ is a Researcher at the Ecole Polytechnique Fédérale de Lausanne (EPFL), Lausanne, Switzerland. From 1997 and 2006, he worked at the Sony Computer Science Laboratory, Paris, on the design of novel approaches to robot learning and on the emergence of cultural systems among machines. He published two books and more than 50 articles in scientific journals, edited books and peer-reviewed proceedings in the fields of epigenetic robotics, complex systems, computational neurosciences, ethology, and evolutionary linguistics.

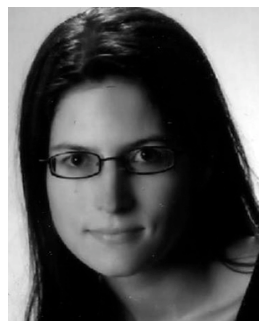

Verena V. Hafner completed her undergraduate studies in mathematics and computer science in Germany, and received the M.Res. degree in computer science and artificial intelligence (with Distinction) from the University of Sussex, Sussex, U.K., in 1999, and the Ph.D. degree in natural sciences from the University of Zurich, Zurich, Switzerland, in 2004.

From 2004 to 2005, she worked as an Associate Researcher in the Developmental Robotics Group, Sony CSL, Paris, France, and joined TU Berlin, Germany, in 2005, as a Senior Research Scientist. Her research interests include neural computation and spatial cognition in the area of biorobotics, and developmental robotics with a focus on joint attention, communication, and interaction. 